\title{
Chérif Ousmane Madani Haïdara et l'association islamique Ançar Dine
}

Un réformisme malien populaire en quête d'autonomie

Cherif Usmane Madane Haydara and the Islamic Movement Ançar Dine. A

Popular Malian Reformism in Search of the Autonomy

\section{Gilles Holder}

\section{CpenEdition}

Journals

Édition électronique

URL : https://journals.openedition.org/etudesafricaines/17056

DOI : 10.4000/etudesafricaines. 17056

ISSN : $1777-5353$

Éditeur

Éditions de l'EHESS

Édition imprimée

Date de publication : 1 juin 2012

Pagination : $389-425$

ISBN : 978-2-7132-2349-5

ISSN : 0008-0055

Référence électronique

Gilles Holder, "Chérif Ousmane Madani Haïdara et l'association islamique Ançar Dine », Cahiers d'études africaines [En ligne], 206-207 | 2012, mis en ligne le 01 juillet 2014, consulté le 28 juin 2022. URL : http://journals.openedition.org/etudesafricaines/17056 ; DOI : https://doi.org/10.4000/ etudesafricaines. 17056

Ce document a été généré automatiquement le 29 septembre 2020.

(c) Cahiers d'Études africaines 


\title{
Chérif Ousmane Madani Haïdara et l'association islamique Ançar Dine
}

\author{
Un réformisme malien populaire en quête d'autonomie \\ Cherif Usmane Madane Haydara and the Islamic Movement Ançar Dine. A \\ Popular Malian Reformism in Search of the Autonomy
}

Gilles Holder

1 Ce texte se veut une contribution à l'étude du mouvement islamique malien appelé Ançar Dine (de l'arabe ansâr al-dîn, litt. ceux qui aident la religion), un mouvement à la fois populaire et controversé qui constitue l'un des principaux espaces de transformation sociale au Mali. Fondé par Chérif Ousmane Madani Haïdara, un personnage hors normes qui a bouleversé le paysage religieux malien et les modalités d'expression de l'islam dans l'espace public, Ançar Dine ne cesse d'attirer un nombre croissant de fidèles et de sympathisants au Mali et dans les pays voisins. Pourtant, si Haïdara et son prosélytisme spectaculaire ont fait l'objet d'études partielles en langue anglaise (Davis 2002; Schulz 2003, 2007; Soares 2005, 2006) et alimentent de nombreux articles de presse au Mali et dans les pays voisins, aucune analyse substantielle n'a encore été entreprise autour du parcours de ce personnage majeur de la vie publique malienne, ni sur la nature de son mouvement iconoclaste.

Cette relative absence d'études sur la forme d'organisation adoptée par Ançar Dine, sa technicité et ses particularités doctrinales est d'autant plus surprenante que ce mouvement religieux est perçu (et requis) comme une plateforme de critiques sociales majeure à l'endroit de l'État et de l'establishment, mais aussi comme la principale alternative islamique face aux dynamiques de ce que l'on appellera le «sunnisme réformé $»^{1}$. On compte aujourd'hui 146 associations et organisations officiellement déclarées (Thiriot 2010), nationales ou internationales, locales ou transnationales, qui toutes participent de l'hétérogénéité de l'islam malien. Celui-ci se singularise toutefois par un faible recrutement confrérique - comparé au Sénégal voisin - et la présence millénaire de « villes bénies » (Holder 2012), telles Tombouctou et Djenné qui rappellent l'ancienneté de l'islam soufi dans l'espace national. Mais si l'islam y est présent depuis 
fort longtemps, le Mali contemporain se caractérise par un phénomène de réislamisation de masse - les chiffres avancés, hors de toute statistique confessionnelle, oscillent entre 90 et $95 \%$ de la population - qui, si elle est relativement récente, confirme néanmoins que la République du Mali, membre fondateur de l'Organisation de la conférence islamique (ocI), est avant tout un pays musulman du point de vue géopolitique.

$3 \mathrm{Au}$ sein de cette sphère islamique dynamique ${ }^{2}$ et hétérogène, quoique majoritairement sunnite et s'inscrivant traditionnellement dans l'école de jurisprudence malékite, une compétition à la fois sociale et spirituelle, voire civilisationnelle, se déploie depuis le milieu des années 1990, laissant un temps hors-jeu les lignages religieux, les saints et autres marabouts qui constituent l'essentiel de la «classe cléricale» (Triaud 1985) malienne. Deux principales tendances, que l'on peut qualifier de "réformistes", s'opposent: d'un côté, les tenants d'un islam littéraliste qui se situent au sein de la réforme néo-hanbalite; de l'autre, ceux qui estiment incarner un islam populaire à travers une intelligibilité culturelle africaine de l'islam malékite, et dont Ançar Dine anime le principal courant aux côtés de mouvements plus récents dits soufis, tels que celui de Cheick Soufi Bilal (Soares 2007, 2010) ou encore celui de Soufi Adama (Soares 2007 ; Bourdarias 2008).

4 Cette compétition, qui anime fortement l'actualité islamique malienne en suscitant des débats au-delà de la sphère religieuse ${ }^{3}$, revêt la forme d'une lutte doctrinale qui s'est longtemps manifestée à travers les pratiques rituelles. Mais elle prend aussi l'aspect d'une lutte historique et personnelle incarnée par deux figures de l'islam malien actuel : Chérif Ousmane Madani Haïdara, le «guide spirituel » malékite de Ançar Dine, et Mahmoud Dicko, l'imam de la mosquée sunnite réformée de Badalabougou et ancien secrétaire général de l'Association malienne pour l'unité et le progrès de l'islam (AMUPI) mise en place par le pouvoir en 1980 pour contrôler les activités religieuses dans le pays (Sanankoua 1991).

5 Si le positionnement sociologique de Haïdara demeure l'islam des sans voix, des illettrés et de ceux qui se voient relégués au ban de la société, depuis l'élection de l'imam Mahmoud Dicko à la tête du Haut conseil islamique du Mali (HCIM) en janvier 2008, puis la contestation qu'il aura su mener à l'égard de la réforme du Code de la famille et des personnes en août 2009 (Soares 2009 ; Thiriot 2010), le rapport de popularité s'est inversé en faveur des sunnites réformés (Anonyme 2009; Meunier 2010). Car, outre la légitimité « naturelle » de ceux qui se réfèrent à un islam censé puiser aux salafí et aux lieux saints de l'Arabie Saoudite, outre le contrôle du bureau du HCIM et outre la manne financière des pays du Golfe dont ils bénéficient, les sunnites réformés concentrent de puissants outils de diffusion à travers des mosquées, des médersas, des médias, des associations et des cercles de réflexion ${ }^{4}$.

6 Mais si l'influence du sunnisme réformé au Mali est massive, l'association Ançar Dine fait, quant à elle, figure d'alternative à cette tentative d'hégémonie salafite et, de ce point de vue, il est pertinent de comprendre la nature de ce mouvement associatif, et d'abord la personnalité de son leader. On doit en effet se demander pourquoi et comment Haïdara, qui a longtemps été perçu par l'establishment comme un fauteur de troubles doté d'une faible instruction religieuse (Schulz 2007 : 41), un agitateur populiste à la tête d'une " secte » (sic) aux visées politiques, est devenu en 2000 secrétaire aux affaires religieuses du HCIM - il en est actuellement second vice-président - et secrétaire aux prêches et aux activités religieuses de la Ligue malienne des imams et érudits pour la solidarité du Mali ( LIMAMA)? Haïdara et son mouvement Ançar Dine semblent de toute évidence être 
devenus incontournables dans le paysage islamique malien, au point que le pouvoir politique y voit désormais, sinon une alternative au sunnisme réformé, tout au moins une force de rééquilibrage susceptible de lui permettre encore de manœuvrer.

\section{Chérif Ousmane Madani Haïdara : un énoncé identitaire comme itinéraire religieux}

7 La genèse du mouvement islamique Ançar Dine, dont le site Internet affirme qu'il compte "plus de 100000 membres, 24 pays, 30 sections, 169 sous-sections, 900 comités $»^{5}$, est étroitement liée à son "guide spirituel » ("dinè nyemògò ») ${ }^{6}$, Chérif Ousmane Madani Haïdara. Aussi, dans un premier temps, nous présenterons les éléments saillants de la biographie de ce leader islamique ${ }^{7}$ qui émerge au milieu des années 1970, en retraçant le parcours par lequel il se construit comme une nouvelle figure de l'islam malien. Dans un second temps, nous évoquerons les caractéristiques de son positionnement doctrinal transgressif qui présente un certain nombre de traits originaux.

\section{Haïdara, ou la genèse d'une vocation}

8 Fils unique de Mamadou Madani et Assitan Traoré, né en 1955 à Tamani, un village situé à soixante-dix kilomètres au sud de Ségou réputé fondé par le conquérant djihadiste al-Hajj 'Umar al-Fûtî (Robinson 1988) à la fin du XIX $x^{e}$ siècle, Haïdara serait resté à l'école coranique de Tamani, dirigée par un certain Bakari Famanta, de 1962 à 1964, tout en suivant l'école française. Le père de Haïdara, affilié à la Tijâniyya, parviendra à négocier le retrait de son fils de l'école pour l'inscrire à la médersa de Saada Oumar Touré de Ségou en 1964.

Louis Brenner (1991b : 73-74) précise que cette médersa, créée en 1946, n'est autre que la toute première à avoir été ouverte par un Malien (avec celle de Kayes). S'inscrivant dans la dynamique qui caractérise alors l'irruption du sunnisme réformé dans l'espace malien (Hamès 1980 ; Amselle 1985; Triaud 1986; Brenner 1991a), Touré s'inspira de cette réforme pour fonder une médersa tijânî qui, aujourd'hui encore, est l'un des plus célèbres centres d'éducation islamique du pays. L'école de Saada Oumar Touré offrait non seulement une alternative doctrinale aux médersas de la réforme sunnite, en conciliant la modernité sociale à une pédagogie tijânî refondée, mais elle se positionnait aussi dans une certaine rupture à l'égard de l'autorité coloniale, laquelle n'a d'ailleurs cessé de lui imposer des restrictions. Or il semble que cette "troisième voie " qui a caractérisé la médersa de Ségou ait profondément marqué les conceptions du futur guide de Ançar Dine, lequel s'érigera peu à peu publiquement comme le principal opposant aux sunnites réformés, tout en maintenant ses distances avec les régimes politiques maliens qui se sont succédé, au moins jusqu'à ces dernières années.

Haïdara quitte la médersa de Saada Oumar Touré en sixième année, courant 1970, lorsque son père souhaite qu'il poursuive sa formation auprès de son ami Bamama Worofana, un maître coranique qui enseignait dans le village de Fasonko, près de Ségou. De l'avis même de Haïdara, le niveau de ce nouveau maître était faible comparé à celui de Touré, au point, dit-il, qu'il ne parlait pratiquement pas l'arabe, même s'il estime qu'il lui a transmis d'autres valeurs. Haïdara demeure à Fasonko quatre ans, jusqu'à la mort de son père en 1974, avant de revenir à Tamani auprès de sa famille et mettre ainsi un terme à sa 
scolarisation religieuse à l'âge de dix-neuf ans. En 1976, il se rend en Côte-d'Ivoire, notamment à Daoukro et Bonkani où il débute ses "prêches » ("wajuli »). En 1978, il revient un an à Tamani, puis part à Abidjan et s'y installe comme "prêcheur » ( "wajulikèla )) et «marabout » ("mòri»). Il rentre définitivement au Mali courant 1981 pour se consacrer alors à la prédication entre Tamani et Mopti. Mais à la suite de graves dissensions avec un prêcheur célèbre qui officiait également à Mopti, Karamogo Lassana ${ }^{8}$, les autorités administratives prononcent la suspension des activités religieuses de Haïdara en 1983.

\section{Haïdara, où la figure exemplaire d'un héros en lutte}

11 Haïdara se rend alors à Bamako chez son demi-frère aîné Yacouba, afin qu'il intervienne pour la levée de cette suspension. Les médiations semblent avoir abouti, car la suspension de Mopti fut levée au bout d'un an. Mais Haïdara n'attendit toutefois pas pour poursuivre sa prédication à Bamako où, à l'invitation de son frère, il aurait été convié à prêcher la nuit à la mosquée de Lafiabougou N'Gabakoro, lors du Ramadan de juin 1984. Eu égard à l'accueil qu'il y aurait reçu, on lui demanda alors de venir prêcher sur l'ancienne place de Railda qui séparait Oulofobougou-Bolibana et Dravéla9 ${ }^{9}$, et sur laquelle officiait un autre prédicateur, lui aussi très populaire, le ségovien Abdoul Aziz Djiré, dont les méthodes de prédication en public semblent avoir inspiré Haïdara (Davis 2002 : 130, 132).

De 1984 à 1989, Haïdara réside successivement dans les quartiers de Quinzambougou, Médina Coura et Ngolonina, ce dernier jouxtant le cœur historique de Bamako qu'est Bozola, tout près des grandes familles maraboutiques Niaré et Touré installées là depuis le milieu du XIX ${ }^{e}$ siècle (Marty 1920a : 65-72). Mais face au succès grandissant des prêches iconoclastes du jeune Haïdara, le Bureau de l'AMUPI de Bamako, au sein duquel la réforme sunnite dominait, réclame une fois encore sa suspension auprès de l'administration et l'obtient à la fin 1989. Haïdara explique sans ambages les motifs de cette suspension, considérant que les mesures de rétorsion dont il fit alors l'objet lui offrent rétrospectivement une légitimité historique, sinon héroïque. Contrairement à celle de Mopti, où le reproche principal portait sur des critiques à l'endroit du pouvoir et de l' establishment, la suspension de Bamako porte sur deux points plus doctrinaux, révélant du même coup que la carrière du prêcheur était en train de passer d'une simple posture de contestation de l'ordre social à une critique de la légitimité du savoir censé justifier cet ordre.

Le premier point reproché se fonde sur une position que Haïdara substantialisera par la suite à travers la formule : «La prière n'est pas l'islam! » ("sali tè silamèya ye »). Au-delà de l'effet de scandale et le risque d'apostasie auquel il s'expose ainsi, Haïdara met en réalité en cause les pratiques islamiques existantes, à travers la critique de la notion de "musulman de naissance » ("silamèden»). Il estime en effet que si la prière est un des piliers de l'islam et une obligation pour tout musulman, celle-ci ne suffit pas pour s'exonérer de vivre en conformité avec le Coran et la charîa. Pour être véritablement musulman, Haïdara considère qu'il faut réaffirmer en conscience son engagement dans le message du Prophète, opérer une sorte de "confirmation », au sens chrétien du terme, qui se concrétise par une série de six "promesses" ("layiduw») qu'il va instituer à travers la pratique de la bay'a, sur laquelle nous reviendrons. Le second point, peut-être moins choquant du point de vue doctrinal, tenait à l'attaque de Haïdara vis-à-vis de la tradition sunnite en affirmant que 'Îsâ (Jésus) est mort et qu'il ne reviendra pas à la « Fin 
du Monde » ("Qiyâmah») pour aider le Mahdi. Accusé de bid'a par l'AmupI, Haïdara se verra donc à nouveau frappé d'une interdiction de prêcher par les autorités politiques fin 1989, décision qui n'était du reste pas sans lien avec la visite pastorale de Jean-Paul II au Mali qui devait intervenir en janvier 1990.

Comme Haïdara le souligne lui-même, qu'il s'agisse de la critique envers ceux qui prient pour simplement se conformer à l'usage social, ou qu'il s'agisse d'affirmer que 'îsâ est définitivement mort, l'important était de faire comprendre qu' il y avait du nouveau dans le prêche à propos de notre islam ${ }^{10}$, c'est-à-dire que quelque chose d'inédit avait été posé à la fois dans l'islam prêché par Haïdara et dans la forme du sermon (Davis 2002 : 131). De fait, s'il insiste sur cette dimension "nouvelle » ( kura»), c'est qu'il prétend dès cette époque être un réformateur qui veut revivifier les pratiques populaires. Et plus que l'incompréhension, c'est le rejet qu'il suscite, notamment auprès des sunnites réformés, puis des imams, des lignages religieux et enfin des autorités politiques, qui explique pour une large part ces suspensions.

\section{Jeux de noms, jeux de titres}

Car quelles que soient ses prétentions réformatrices, et au-delà de son opposition à la réforme sunnite, Haïdara apparaît en effet comme un phénomène incontrôlable dans ce monde largement coopté des élites religieuses maliennes. Aussi, dès son irruption sur la place de Railda, on met en cause non seulement le niveau de ses connaissances, mais aussi ses origines jugées obscures et l'identité chérifienne douteuse de cet inconnu donneur de leçons. De fait, l'énoncé identitaire «Chérif Ousmane Madani Haïdara » par lequel il se présente aujourd'hui est le résultat provisoire et conjoncturel d'une construction que l'intéressé ne cesse de réviser, et sur laquelle il s'agit de s'arrêter brièvement.

$\mathrm{Si}$, au Mali, il peut a priori sembler légitime de prendre le titre de chérif lorsqu'on porte le nom chérifien de Haïdara, pour le cas d'espèce, il s'agit d'un nom tutélaire que l'intéressé aurait hérité d'un certain Chérif Moussa, tenu comme étant l'ancêtre familial et dont il vénère la tombe chaque année à Djédabougou Wéré, près de Tamani. Pire encore, la rumeur prétend que le nom Haïdara aurait été donné à sa famille comme équivalent islamique à celui de Traoré, réduisant dès lors sa filiation à une vulgaire identité « marka » qui se réfère à un peuplement historiquement singularisé par des liens avec le Mali médiéval, la pratique du commerce et l'islam. De leur côté, les partisans de Haïdara défendent évidemment son origine chérifienne. Certains le rattachent au lignage de Sidi Ibrahim Haïdara, plus connu sous le nom Nyaro Karamogo, l'un de ces modestes «hommes de religion» (Triaud $1985: 273$ ) de la région de Ségou qui prêcha en milieu paysan durant les années 1920-1930 ${ }^{11}$. Mais la genèse officielle des chérifs de Tamani mise en lumière par Ançar Dine est celle d'une origine iraquienne qui, dans la phase malienne du parcours, passe par Djenné (ou Dia) et la mise à l'épreuve de leur sainteté - plonger la main nue dans l'huile bouillante pour récupérer la bague du roi -, avant de s'installer à Tamani il y a environ deux cents ans et y exercer alors modestement leur magister. Cette genèse officielle précise en outre que si l'on peut porter le nom Haïdara sans être chérif, notamment pour ceux qui se réclament de la descendance de 'Alî sans passer par ses fils Husayn et Hasan, l'inverse est impossible. Dès lors, l'emploi simultané des noms-titres chérif et Haïdara est une manière pour le guide de Ançar Dine d'affirmer sa pleine légitimité chérifienne en dépit des contestations. 

on peut le devenir» (Rhani 2009: 32), pour peu que l'on bénéficie de la baraka qui singularise le saint, ou plus exactement le « rapproché de Dieu » («walî»). Et Rhani de rappeler que «n'étant pas tous à l'origine des chorfa, des saints et leurs descendances ont tenté par tous les moyens de s'insérer dans la lignée prophétique, soit en obtenant un décret sultanien légitimant cette appartenance, soit par le témoignage du prophète luimême qui apparaît en songe et certifie la réalité de la filiation » (ibid.). S'agissant de Haïdara, l'autorité politique n'a évidemment délivré aucun décret. Sa légitimité chérifienne est donc plutôt à rechercher du côté de la grâce divine et de la validation populaire d'une trajectoire religieuse iconoclaste.

\section{Haïdara, le chérif absolu}

Si les détracteurs de Haïdara n'apportent pas de preuve tangible quant à la dénégation de son titre chérifien, la « rumeur» («mògòkan») tend à diffuser le fait que, du prestigieux énoncé identitaire, il ne resterait au prêcheur que le nom de son père : Madani. De fait, dans un récent article de presse, par ailleurs complaisant et autorisé ${ }^{12}$, on apprend que Haïdara était appelé Chérif Bazoumana dans son village, du nom d'un ancien muezzin de la mosquée de Tamani. Ce n'est que lorsqu'il intégra la médersa de Ségou que son maître Saada Oumar Touré lui révéla que Bazoumana n'était autre que la prononciation en langue bamanan de Ba Ousmane, c'est-à-dire « Vieux Ousmane ». Ni chérif, ni Haïdara, ni tout à fait Ousmane, celui qui deviendra le prêcheur le plus célèbre du Mali, celui que les médias qualifieront de «mouride malien» ou d'« imam rouge » (Serge Daniel de RFI), celui enfin que ses partisans surnommeront simultanément Bani (Le Descendant) ${ }^{13}$, Wulibali (L'Incontestable) ou Nyemògò (Le Guide), est d'abord un self-made man qui recrute avec d'autant plus de succès qu'il incarne une figure de la réussite pour tous ceux qui n'héritent de rien. Aussi, Haïdara balaie-t-il d'un revers de main ces mises en cause autour de son identité visant sa légitimité religieuse: «Puisqu'on nous fatigue avec le nom Haïdara », déclare-t-il au journaliste qui l'interroge à ce sujet, « moi je veux devenir Coulibaly, pour mieux faire mon travail. Un homme doit se glorifier à travers les actes qu'il pose et non vivre sur le nom de ses parents. En devenant Coulibaly je serai à l'aise » (Traoré 2007).

19 La réplique est pleine d'impertinence, car Coulibaly évoque le nom commun par excellence au Mali - sorte de Monsieur Dupont - et est notamment requis dans le cadre des « relations à plaisanterie ritualisées » (" sanankuya »). La réponse est surtout cinglante à l'endroit des aristocraties religieuses qui se targuent d'être les garants d'un islam installé et validé au nom d'une tradition et d'un héritage que Haïdara considère de peu de valeur; pour lui, le message prophétique est d'agir sur le monde d'ici-bas autant que d'envisager son propre salut dans l'au-delà. Par cette position intramondaine, Haïdara assume ici sa propre construction identitaire, y compris lorsque celle-ci est ambiguë. C'est du reste tout le sens de l'effort qu'il déploie depuis 2006 pour fixer sa biographie, à travers une stratégie scripturale et plurilingue qui indique qu'il souhaite désormais aller au-delà des supports audiovisuels qui ont caractérisé son prosélytisme populaire.

C'est ainsi qu'en 2009, à l'occasion de la diffusion de son prêche du Maouloud, il substitue son nom Chérif par le titre "Aséid » sur la pochette de ses vidéos-CD, révélant en cela qu'il considère bien le nom Chérif comme un titre. Il devient alors «Aséid Ousmane Madani Haïdara » puis, en 2010, « Aséîd Chérif Ousmane Madani Haïdara ». Si ce nouveau 
titre n'est pas sans ambiguïté, puisqu'il est pris par nombre de ses fidèles comme signifiant simplement «cheick », cet emprunt au registre persan du titre as-Sayyid, quasi équivalent au terme arabe Sharîf, constitue une réponse insolente à l'endroit de ceux qui lui contestent l'usage du terme chérif. Non seulement parce qu'il signifie qu'il est un descendant des chérifs de La Mecque, mais parce que sa dernière innovation biographique se veut une référence implicite au monde iranien, voire chiite ${ }^{14}$. Enfin, son nouveau titre Aséïd n'est rien d'autre que la réaffirmation d'une légitimité religieuse qu'il estime ne devoir tenir que de ses seuls partisans, les fidèles de son mouvement d'abord, "ceux de Ançar Dine» ("ansaardinuw»), mais aussi ceux bien plus nombreux qui affirment qu'il dit la « vérité » (« tiyèn ») et qui renvoient à « ceux qui écoutent Haïdara » (" haydara lamènbaaw»).

Sur le même registre, la critique de Haïdara envers les héritiers est aussi une mise en cause de la légitimité de la transmission de la baraka et du statut de saint, de «rapproché de Dieu " («waliju», de l'arabe walî), ce qui ne l'empêche d'ailleurs pas de se laisser qualifier de " cheick » par ses fidèles et d'être bienveillant à l'égard de ceux qui le louent, sinon pour ses « miracles » (« kabako», de l'arabe karâma), du moins parce qu'il est « celui qui a un destin » (" nankama»). En effet, contrairement aux aristocraties religieuses et aux cheicks des confréries historiques, Haïdara ne revendique pas de baraka héritée, mais une baraka originelle directement transmise par le Prophète, ce dont est censé témoigner son nom-titre de Chérif et le parcours de sa réussite spirituelle. De même, dans sa biographie officielle, il ne cherche pas à établir une liste de miracles, à l'instar d'un Cheick Soufi Bilal (Maïga s.d. : 44-45). Prenant à la lettre son surnom de Bani, qui ne le fait pas seulement chérif mais littéralement un descendant des chérifs de La Mecque, Haïdara se présente comme une sorte de saint sans miracle, ou plus exactement comme un homme dont la vie est, à l'image du prophète de l'islam, un miracle en soi par le fait qu'il «transforme la société » ( "ka yèlèma naa ti sigida kònò »). Son laudateur attitré, Nouhoum Dembélé, a ainsi popularisé l'œuvre de "réislamisation » de Haïdara dans un chant intitulé Folikan (litt. Salutation) ${ }^{15}$, chant qu'il a adapté en 2004 à partir de la célèbre chanson éponyme créée en 1988 par l'Orchestre régional du Super Biton de Ségou ${ }^{16}$ en l'honneur de l'ancien président Moussa Traoré. Véritable panégyrique officiel du réislamisateur Haïdara, où il y est présenté comme celui qui « a pris le Coran pour le donner aux Musulmans ", ce chant est aussi devenu l'« hymne de l'Ançar Dine Internationale $»^{17}$.

Dès lors, l'ennemi historique des sunnites réformés apparaît à son tour comme un réformateur qui ne cesse de remettre en cause la légitimité des lignées maraboutiques et autres saints vivants, mais aussi un réislamisateur révélant l'islam vrai. S'efforçant de pondérer les tentatives répétées faites par ses fidèles de le présenter comme un saint sans toutefois les en dissuader totalement -, il s'attache à capitaliser son parcours religieux comme un modèle de vie sainte à l'image de celle du prophète Muhammad. Mais l'autoproduction de cette figure majeure de l'islam malien repose aussi sur la construction en parallèle d'un mouvement qui va prendre forme entre 1984 et 1989, Ançar Dine, relatée sur le mode de la geste muhammadienne.

\section{Ançar Dine : entre épopée muhammadienne et association de la société civile}

En s'installant sur l'ancienne place de Railda en 1984, Haïdara semble avoir été bien accueilli, au point de détrôner le célèbre prêcheur Abdoul Aziz Djiré qui officiait avant lui 
sur les lieux. On rapporte que les deux maîtres se seraient livrés à une véritable joute publique fin 1984 début $1985^{18}$, où Haïdara aurait argué que l'usage des mèches par les femmes était réprouvé par l'islam, non seulement parce qu'elles se signalaient de façon indécente en suscitant le désir chez les hommes, mais surtout parce que les mèches empêchaient la réalisation conforme des ablutions de la tête. Abdoul Aziz Djiré aurait répliqué que l'on ne peut tout interdire et argumenté sur le fait que les mèches n'altéraient pas les ablutions. C'est pourtant la rhétorique moralisante de Haïdara qui va prévaloir dans cette joute, au point qu'un groupe de six femmes le sollicitera afin qu'il continue de prêcher, en lui promettant en échange de l'aider « à cause de Dieu et de son prophète » (Traoré 2007 : 14).

\section{Haïdara, le « marabout des femmes »... à l'imitation du Prophète} tournant des années 2000 par de jeunes prêcheurs que l'on appelle les « Haïdara juniors ", est non seulement de parler essentiellement des femmes, de leur comportement, de la nécessité du mariage ou de leurs tenues vestimentaires, mais aussi d'être principalement financés et aidés par les femmes. D’où le surnom de «marabout des femmes » (« musow ka mòri ») dont Haïdara s'est vite vu affublé par ses détracteurs ${ }^{19}$. D'un point de vue social, les femmes se font ainsi les promotrices des agents de moralisation à leur endroit, tout en se constituant en public privilégié d'un spectacle religieux qu'elles contribuent à produire. Ce faisant, et au prix du port d'un voile lâche pour les femmes d'un certain âge, attaché pour les jeunes femmes - là où les sunnites réformés requièrent quant à eux le voile intégral -, elles échappent en partie à la tutelle des pères et des époux qui ne peuvent s'opposer à ce qu'elles rejoignent leur prêcheur. En participant matériellement à ces prêches, les femmes s'émancipent et s'érigent en actrices de la chose publique qui, au Mali, porte la légitimité la plus forte : celle de la religion islamique.

dèle muhammadien toujours, puisque la promesse de ces six femmes s'engageant à soutenir l'œuvre de leur prêcheur aurait donné lieu à la première mention de la bay ${ }^{6} a^{20}$, tirée du verset 12 de la sourate 60 Al-Mumtahanah, "L'Éprouvée ». Ce serment des croyantes au Prophète rapporté par le Coran est connu sous le nom de « Premier Serment d'Allégeance à Al-'Aqaba ", effectué en 621 par douze Ansârî de Yathrib, dont cinq des six premiers hommes qui s'étaient rendus à La Mecque l'année précédente et avaient embrassé l'islam. Nous reviendrons plus en détail sur cette question de la bay'a, mais il est intéressant de souligner que c'est par un tel serment que Haïdara identifie rétrospectivement la genèse de son mouvement. Il s'agit de mettre ici en scène les six premières femmes qui s'engagèrent autour de Haïdara, articulant ainsi le prototype féminin de Khadîja, première épouse du Prophète et première musulmane, aux six premiers Ansârî convertis, tandis que le biographe réintroduira par la suite le soutien des 
premiers hommes au prêcheur (Traoré 2007: 15), afin de mettre la narration en conformité avec le serment de Al-'Aqaba.

En réalité, la fixation du récit de cet engagement des premiers partisans s'effectue au milieu des années 2000, faisant correspondre ce moment crucial qui s'étend sur les cinq années séparant les deux interdictions de prêche de Haïdara aux cinq premières années prophétiques qui virent la "Secte des Musulmans» atteindre une centaine de membres. Et c'est donc durant cette période que, selon Haïdara lui-même, ses partisans auraient émis le souhait d'appeler leur mouvement "Ceux qui aident Haïdara " (" haydara dèmè bagaw»). Il leur aurait alors demandé de l'appeler plutôt "Ceux qui aident Dieu » (" ala dèmè bagaw») et "c'est ainsi, explique-t-il, que Ançar fut créé » ${ }^{21}$ comme cadre d'une réforme islamique malienne en référence aux soutiens médinois du prophète Muhammad ${ }^{22}$.

\section{Ançar Dine, où le récit d'une réforme populaire hégirienne}

De fait, Ançar Dine se constitue bel et bien comme un mouvement réformiste, moins visà-vis du dogme, qui reste fidèle au sunnisme et au malékisme, que dans sa forme associative conforme à la nouvelle société civile (Schulz 2003 : 160). Haïdara à la tête de Ançar Dine emprunte à la fois à la geste muhammadienne et à l'image populaire du guide confrérique (modestie, vérité, piété), tout en s'affranchissant du "serment d'affiliation » à un cheick (bay'a ou mubay' $a$ en arabe), des « retraites spirituelles » («kaluwa », de l'arabe khalwa) et des «miracles» ("kabako»). Quant au savoir religieux qu'est censé détenir le guide d'un tel mouvement, objet de suspicions à son égard de la part des élites islamiques, son relatif déficit est un élément de légitimité, car Haïdara s'appuie ici encore sur l'exemple du prophète Muhammad qui, réputé illettré, révèle un message divin qu'il n'aurait pu concevoir en se fondant sur son seul savoir. De fait, s'il n'est pas illettré, Haïdara revendique une formation limitée à une dizaine d'années d'étude et apparaît donc d'une certaine manière comme le Prophète, plus inspiré que savant, et porteur de la vérité fondamentale du message divin. C'est d'ailleurs en ce sens que le réformisme du guide de Ançar Dine est un fondamentalisme, en se voulant lui aussi un rappel aux « pieux ancêtres" («salaf sahîh» en arabe), mais fondé ici sur une spiritualité résolument populaire. Enfin, comme le prophète de l'islam, Haïdara est en lutte contre les autorités religieuses et politiques de son époque ${ }^{23}$, lesquelles le contraindront à quitter Mopti comme s'il avait dû partir de La Mecque pour s'installer à Bamako avec ses Ançars, comme s'il était entré à Médine.

Cette geste hégirienne, le mouvement Ançar Dine va la rejouer encore lorsque, à la fin de l'année 1989 où il vient à nouveau d'être interdit de prêche, Haïdara quitte le centre-ville de Bamako pour s'installer en périphérie dans le quartier non viabilisé de Banconi, à Djanguinèbougou. Haïdara a alors 34 ans et acquiert pour la première fois une propriété, marquant ainsi son ancrage en un lieu qui lui permet de construire son logement et une petite mosquée de fondation, un moment fort que les fidèles nomment "Chantier de Banconi » («bankoni santie ») et qui fait figure d'hégire locale (Bourdarias 2008 : 123).

Haïdara va acquérir peu à peu les terrains adjacents pour y loger un certain nombre de fidèles et accueillir les structures du mouvement qui ne cesse de croître. S'il fait construire le siège social de l'association à partir de 1992-1993, suivi de quelques autres modestes réalisations, c'est surtout à partir de 2004-2005 que l'urbanisation « en dur » va débuter, selon un schéma directeur révélant les priorités du mouvement. Il fait ainsi bâtir 
successivement un groupe scolaire qui dispense un enseignement allant du niveau fondamental au lycée (2005-2006 avec extension en 2010-2011), le siège social de Ançar Dine International (2006 avec extension en 2009), un bâtiment de logistique pour le Maouloud (2006-2007), un vaste immeuble pour lui et sa famille ${ }^{24}$ (2008), un centre de santé conventionné (2008-2009), une maison des hôtes (2010), une mosquée du vendredi (2010-2011), etc. Preuve de l'importance de cette implantation urbaine s'il en est, les autorités ont dû goudronner la rue principale menant à ce quartier. Officiellement, cette décision serait justifiée par l'existence du centre de santé. Mais elle l'est tout autant par l'importance de Ançar Dine et l'affluence du quartier, surtout au Maouloud où plusieurs dizaines de milliers de fidèles venus de tout le Mali et de la sous-région se pressent, le quartier prenant alors des aspects de souk arabe.

Cette imitation de l'hégire est bien évidemment un récit rétrospectif qui vise à remettre en ordre ce qui, pendant longtemps, a été une aventure pour le moins erratique. Mais le fameux « Chantier de Banconi » n'est pas seulement l'aboutissement en forme d'hégire du parcours de Haïdara, où le guide et ses Ançars s'attachent désormais à faire surgir, au cœur de Bamako, une cité sainte qui évoque Médine, à l'instar de Touba pour les Mourides du Sénégal (Gueye 2002: 84 sq.). Le "Chantier de Banconi» c'est aussi et d'abord le temps de la création de l'Association musulmane pour le soutien de l'islam ( AMSI) en 1991, au lendemain de la chute du régime dictatorial de Moussa Traoré, permettant alors d'inscrire Ançar Dine dans le champ légal des associations de la nouvelle société civile ${ }^{25}$.

\section{Ançar Dine : du mouvement « spontané » à l'association internationale}

32 En dépit du fait que Haïdara s'était installé à Banconi, le siège de l'association fut initialement fixé à Sikasso, riche métropole malienne du sud-est du pays et héritière d'une prestigieuse histoire de résistance à la conquête coloniale. Cette ville avait été préférée à Bamako, où Haïdara était tenu d'être discret depuis les recommandations de la Conférence nationale rejetant la perspective de partis religieux auxquels aspiraient alors les cadres de l'AMUPI.

En réalité, Sikasso concentrait une série d'avantages pratiques pour l'association. C'est d'abord sa forte hétérogénéité religieuse entre musulmans, chrétiens et pratiquants des " cultes à initiation " ( "jow»), laissant ainsi du champ à l'installation de Ançar Dine dans la région. C'est également parce que Sikasso abritait de nombreux fidèles de Haïdara, originaires surtout de Ségou et Bamako, où ils exerçaient une certaine influence; c'est d'ailleurs de Sikasso que provenait la majeure partie de l'aide matérielle que Haïdara reçut durant sa suspension à Bamako. C'est enfin parce que Sikasso se situe à proximité de la Côte-d'Ivoire, sur la route de Bouaké qui fut la principale destination des migrants maliens jusqu'à la crise ivoirienne de 2002.

On le voit, cette position géographique offrait non seulement l'avantage d'être un carrefour propice au prosélytisme du mouvement, mais il était aussi un centre économique des plus dynamiques du Mali, en même temps qu'une éventuelle issue si le pouvoir malien avait pris des mesures de rétorsion à l'égard de Ançar Dine. 


\section{Une association nationale territorialisée et un guide spirituel}

De 1991 à 1992, AMSI s'organise dans un cadre national sur la logique territoriale du parti de l'indépendance US-RDA : le comité au niveau du village ou du quartier ; la sous-section au niveau de l'arrondissement; la section au niveau du cercle; le bureau régional à l'échelle de la région (qui sera supprimé par la suite) ; enfin le bureau national implanté à Sikasso et présidé par Haïdara lui-même. Cette couverture du territoire national à travers une organisation pyramidale est inédite dans ce contexte islamique, hormis pour l'AMUPI, bien que l'espace d'extension de Ançar Dine n'aie jamais été au-delà de la zone linguistique mandingue et bamanan, c'est-à-dire jusqu'au niveau de Mopti. Et aujourd'hui encore, si le mouvement poursuit son extension et sa densification, il peine à s'implanter au nord et à l'est du Mali, où les élites islamiques parlent arabe et/ou hassânîya.

Lors du $1^{\text {er }}$ congrès de l'association, daté de 1992, un bureau national fut élu, présidé par un jeune enseignant de médersa malien, Abdoulaye Diawara. La conjoncture politique se normalisant, c'est à cette occasion que la décision fut prise de transférer le siège de AMSI à Bamako. Parallèlement, prenant en compte le fait que de nombreux fidèles étaient liés à la migration malienne et installés dans les pays limitrophes, la Côte-d'Ivoire et le Burkina Faso notamment, le bureau national décida de créer des bureaux nationaux dans les trois pays et de faire siéger les présidents de ceux-ci au sein du bureau malien. Mais très vite, face au développement de l'organisation hors du Mali, fin 1995-début 1996, AMSI mit en place une coordination des bureaux nationaux qui, en janvier 2004, devint une structure baptisée Ançar Dine international (ADI), constituée des trois bureaux nationaux du Mali, de Côte-d'Ivoire et du Burkina Faso, qui prirent quant à eux le nom de Ançar Dine national $(\mathrm{ADN})^{26}$.

Dès 1992, le statut de " guide spirituel » avait commencé à être évoqué pour définir le rôle de Haïdara au sein du mouvement, statut qui ne sera toutefois entériné que bien plus tard, puisque le règlement intérieur de 2001 ne mentionne encore que le titre de « Grand prédicateur" (Davis 2002: 137). Des dissensions en termes de leadership étaient rapidement apparues entre Haïdara et le bureau autour de la distinction entre la structure associative et son chef religieux, témoignant de la sorte de l'influence croissante des non-Maliens qui défendaient le principe de la séparation en arguant de la nécessité, fallacieuse ou pas, de "protéger " Haïdara des affres de l'association. La décision de "sortir » Haïdara du bureau fut adoptée lors du $2^{\mathrm{e}}$ congrès, fin 1995-début 1996 ; ni président, ni membre du bureau, Haïdara sera désormais simplement consulté pour valider les décisions de l'association et donner sa caution morale. Des modifications des statuts et du règlement intérieur vont avoir lieu en 2002, 2006 et surtout en 2008 et $2009^{27}$, visant à entériner la place centrale de l'ADI dans le dispositif et préciser la position de Haïdara. L'article 11 des statuts de 2008 définit ce dernier en tant que « garant moral et spirituel de l'ADI » veillant à «la normalité et au respect des orientations politiques définies par la Conférence Internationale de l'ADI », l'organe suprême du mouvement et au sein duquel il siège. En outre, selon l'article 3 du règlement intérieur de 2009, le guide spirituel, quoique extérieur à l'organe de décision de l'ADI, « dispose d'un droit de véto et d'interpellation de tous les organes de l'ADI. Il tranche en dernier ressort toute divergence ou tout conflit né au sein de l'ADI ». 


\section{Ançar Dine international : une association islamique « fédérale »}

islamique qui est de s'être développé, non pas dans une perspective transnationale, mais internationale, en ce sens où l'espace pertinent dans lequel il se définit est celui des ÉtatsNations. Si cette logique internationaliste est à l'origine empirique, conjuguant le tropisme économique ivoirien et l'espace linguistique mandingue, elle n'en reste pas moins constitutive de la dynamique institutionnelle du mouvement et va jouer un rôle central dans le devenir de celui-ci.

Nous avons signalé plus haut que le modèle d'organisation territoriale de Ançar Dine était celui de l'US-RDA, le parti de l'Union soudanaise - Rassemblement démocratique africain dirigé par Modibo Kéita, qui a théorisé l'indépendance nationale du Mali et contrôlé la république jusqu'en 1968. Panafricaniste et défendant le principe d'un socialisme appliqué aux réalités africaines, le parti unique Us-RDA s'est singularisé à l'époque par un effort de synthèse entre construction de l'État-Nation et fédéralisme. Ce n'est pas ici le lieu de rappeler les déboires de ce panafricanisme et la fin de la Fédération du Mali, mais pour Ançar Dine, l'us-RDA reste la référence populaire d'une indépendance nationale fondée sur l'intégration régionale et des valeurs africaines dont il se veut le promoteur. De fait, si la rhétorique politique de l'US-RDA est relayée par la référence islamique, le contenu identitaire et culturel est repris dans le projet de Ançar Dine. Ainsi, l'ultime modification des statuts en 2008 confirme ce fédéralisme en définissant l'ADI comme association internationale de droit malien intégrant «les associations, mouvements et fondations se réclamant du cheikh Chérif Ousmane Madani Haïdara, Guide spirituel des Ançars [...] conformément à la Déclaration Universelle des Droits de l'Homme » (art. 1). Se donnant comme but suprême le soutien du guide spirituel dans son « enseignement et la diffusion, à travers le monde, d'un islam vrai fondé sur les préceptes du saint Coran et la Sunna [...] » (art. 4), l'ADI a procédé à une véritable réorganisation internationalisée, avec une structure de pouvoir complexe qui n'est pas sans évoquer la bureaucratie étatique : «Le Guide spirituel; la Conférence Internationale (cI) du Maouloud; le Conseil International des Présidents (CIP); la Trésorerie Internationale (TI); le Comité International de Contrôle (cIc); le Comité International du Woudouhou (cIW); le Secrétariat Exécutif (SE) » (art. 13).

Cette réorganisation marque d'une certaine manière la fin du tropisme organisationnel malien, et peut-être aussi une pondération du charismatique au profit de réformes institutionnelles jugées plus "modernistes" (sic), où l'ADI faisait déjà suite à une ONG fondée en 1994 appelée Mouvement Ançar Dine (MAD). L'ADI contrôle désormais l'ensemble du dispositif, avec un président ivoirien élu lors du Maouloud 2011. Quant aux ADN, si elles disposent d'une relative autonomie d'organisation, c'est dans la limite où leurs spécificités demeurent conformes aux statuts et règlement de l'ADI.

Mais pour internationale qu'elle soit, cette organisation n'est pas qu'une structure impersonnelle. En mettant à profit les facilités de circulation au sein des espaces économiques de la CDEAO et de l'UEMOA ${ }^{28}$, l'ADI aura renforcé l'idée d'une appartenance à une communauté d'hommes et de femmes qui se reconnaissent comme Ançars. On peut être frappé par l'émergence de ce qu'il convient sans doute d'appeler un «peuple » qui éprouve le sentiment de plus en plus puissant de constituer une organisation où les spécificités historiques des ADN sont préservées, où les statuts de l'ADI font office de

Cahiers d'études africaines, 206-207 | 2012 
constitution fédérale et où, comme le remarquait Davis (2002: 140-141), la carte de membre est tenue pour une carte d'identité.

Par sa cohérence territoriale et culturelle, l'ADI réactive une sorte de synthèse entre fédéralisme territorial et fédéralisme communautaire qui n'est pas sans évoquer le projet de l'US-RDA, mais que l'on doit comprendre désormais comme l'expérience en cours d'une umma ouest-africaine. Toutefois, c'est du contrôle des orientations religieuses, enjeu stratégique autant qu'économique, dont dépend le devenir de cette umma et, de ce point de vue, une association sans récépissé de déclaration hébergée en son sein, le Whoudouh ou Woudouhou (de l'arabe hudu, direction), semble poser problème à l'ADI.

\section{Le Whoudouh, ou la bonne « direction » de Ançar Dine}

Créé en 1992 afin de parer à une éventuelle dissolution de AMSI, le Whoudouh a très tôt été conçu comme une officine définissant les orientations doctrinales du mouvement, d'où son nom la «[bonne] direction ». Le rôle principal du Whoudouh, qui regroupe presque un millier de prêcheurs et qui se structure au niveau des trois ADN, consiste à contrôler les activités de Ançar Dine par un travail sur tous les contenus diffusés, hormis pour les productions des chanteurs-louangeurs proches de Ançar Dine et qui, en tant qu'artistes, ne sont pas (encore) assignés à un contrôle très strict. Le Whoudouh est ainsi chargé de la formation des prédicateurs qui leur délivrent un "quitus de qualification ». Depuis 2004, il les réunit à Bamako pour un "séminaire de formation des prédicateurs " qui se déroule durant la semaine du Maouloud. Le Whoudouh élabore également les consignes de prêche, les sourates à mettre en avant et leurs commentaires qui doivent se référer aux hadîth "sacrés» ("qudsî») et "authentiques » ("sahîh») pour éviter toute critique. Enfin, il conçoit les manuels pédagogiques, ou « leçons ançar » ( ansar walanda ») , destinés aux élèves des médersas du mouvement. Mais à mesure que l'ADI étend sa structure bureaucratique, le Whoudouh devient l'instrument par lequel Haïdara maintient un contrôle à la fois doctrinal et financier sur l'ADI, les membres du Whoudouh étant statutairement définis en tant que "représentants " ("lasigiden ») de sa guidance (Davis $2002: 157$ ).

Cette autonomie du Whoudouh n'est pas sans poser problème, et certains hauts cadres de l'ADI considèrent que les prêcheurs parasitent le mouvement en profitant de leur filiation spirituelle à Haïdara pour s'enrichir et faire carrière. L'autonomie du Whoudouh a ainsi été remise en cause dès 2008, avec la suppression des cotisations versées par les prêcheurs pour financer leur propre association. La révision du règlement intérieur du 10 mars 2009 a confirmé cette tendance avec la création d'un Comité international du Whoudouh (cIW) intégré à l'ADI. Une série d'articles du règlement précise ce contrôle sur les prêcheurs qui doivent désormais avoir été «membres actifs» d'un ADN pendant au moins deux ans (art. 26), se plier aux décisions du Conseil international des présidents ( CIP) qui pilote l'ADI (art. 27), «se soumettre à l'autorité de la structure nationale dont ils sont originaires » et "travailler sous la direction des conseillers chargés des questions religieuses » (art. 28) de l'ADI.

Si cette refonte montre un rapport de force favorable à l'ADI aux dépens des charismatiques, elle indique aussi le renforcement à la fois de la bureaucratisation du mouvement et de la dimension doctrinale dans sa gouvernance. Mais au-delà, l'enjeu essentiel de cette reprise en main du Whoudouh par l'ADI a trait à ce qui constitue un 
véritable tabou interne : l'évolution du mouvement et l'anticipation de l'après-Haïdara. La question a récemment encore été soulevée lors de l'Assemblée internationale du 19 février 2011 et la réponse du nouveau président de l'ADI a été cinglante : « Pour ce qui est de la question de remplacer le maître par l'un de ses fils, je ne veux plus jamais entendre quelqu'un parler de cela. Cette décision ne revient qu'au maitre lui-même qui est libre de se faire remplacer par l'un de ses fils au moment qu'il aura choisi ! » Le mouvement vit en effet un moment crucial quant à son organisation. L'alternative se situe entre le développement de la structure bureaucratique et la transformation en un mouvement de type confrérique. Selon l'option choisie, Haïdara aura alors à repréciser les modalités de transmission de sa guidance définies par les statuts, en l'occurrence à l'un de ses fils (art. 12), tandis que l'ADI devra se déterminer entre la forme transnationale, à l'instar du modèle confrérique qadirî ou tijânî, ou bien fédérale, inspirée du modèle plus politique de l'État-Nation porté jadis par l'Us-RDA.

\section{L'argent du culte : entre cotisations et bénédictions, un projet de banque islamique}

L'une des caractéristiques de Ançar Dine serait, dit-on, son autofinancement (Davis 2002 : 142), argument dont le mouvement se prévaut à l'encontre de la mouvance sunnite réformée, connue pour être aidée par les pays du Golfe, l'Arabie Saoudite en tête. Cela ne signifie évidemment pas que Haïdara ne puisse recevoir des cadeaux et dons financiers à titre personnel, que ce soit de la part de fidèles ou d'individus liés à certains pays musulmans - Libye et Iran notamment -, mais aussi de l'État malien, par l'intermédiaire de la personne du président de la République ou de son épouse. Mais en tout état de cause, ces fonds sont quasiment impossibles à chiffrer de l'extérieur, et ce d'autant que Haïdara en réinjecte une partie au profit de la réalisation des infrastructures du mouvement, de sociétés économiques diverses liées à l'action prosélyte, et qu'il effectue des dons à titre privé aux sections ançars qui le sollicitent.

Théoriquement très encadrées, les ressources officielles de Ançar Dine sont fixées par l'article 14 des statuts de 2008 et les comptes vérifiés par le Comité international de contrôle (cic) qui a «obligation de présenter un rapport annuel à la Conférence Internationale à l'occasion de chaque Maouloud » (art. 24). La première source de revenus du mouvement est une cotisation annuelle dite « dépense pour l'année » ("san musaka »), qui s'élève à 1000 FCFA par mois et par membre, soit 12000 FCFA par an perçus par les sections et dont l'intégralité des montants est envoyée à l'ADI. Il y a ensuite le " paiement mensuel » (« kalo sara »), qui s'élève à 500 FCFA par membre, dont 200 FCFA sont envoyés au siège de l'ADN, tandis que les 300 FCFA restants vont à la caisse de la section pour financer les campagnes de prêche locales, l'entretien du siège, le soutien aux membres nécessiteux, etc. Selon les responsables de l'ADI, seuls $50 \%$ des quelque 70000 membres recensés en 2009-2010 versent leurs cotisations annuelle et mensuelle, contribuant ainsi à un total que nous avons évalué entre 620 et 630 millions de FCFA.

Au-delà de ces cotisations, il existe des ressources provenant de deux types d'activité. Il s'agit des fonds, issus soit d'une activité économique soit de dons personnels, qui réfèrent à une économie morale particulièrement valorisée que l'on appelle respectivement «travailler à cause de Dieu » (" ka baara kè ala kama ») $)^{29}$ et « donner à cause de Dieu » (" ka di ala kama»). Les sommes réunies dans le cadre de cette économie de la cotisation, du travail et du don font l'objet d'une «bénédiction » («dubabu», de l'arabe du'â', «prière 
personnelle, supplication ») prononcée publiquement par Haïdara au Maouloud. Celle-ci est considérée comme le moment fort de la cérémonie, où il s'agit de conversion en baraji (pour l'au-delà) et en baraka (pour l'ici-bas). En dépit de la variabilité de ce type de ressources, le montant global atteindrait au moins 400 millions de FCFA par an, qui s'ajoutent aux 620 ou 630 millions des cotisations, donnant un total d'un peu plus de 1 milliard de FCFA annuel ${ }^{30}$.

49 Pour valider cette estimation budgétaire, nous avons évalué les dépenses effectuées en 2009-2010 par l'ADI, qui s'élèvent à environ 1,5 milliard de FCFA par an réparties entre les cérémonies religieuses, la construction d'immeubles et les frais de fonctionnement de la structure. Une partie marginale des 500 millions manquants est peut-être issue de dons de gré à gré, tandis qu'une autre faible partie proviendrait des activités de l'ADI, dont la commercialisation des enregistrements des prêches, la vente des produits dérivés, l'agence de voyage Chérifla dédiée au hajj, etc. Mais l'essentiel de ces 500 millions de FCFA est issu de l'épargne constituée les années précédentes, prévue pour pallier notamment les difficultés à accéder à des prêts immobiliers aux taux d'intérêt prohibitifs (entre 13 et $18 \%$ annuels), principal obstacle à l'autonomie financière d'un mouvement qui peine à aller au-delà de cet autofinancement.

En effet, l'organisation internationalisée, le nombre de fidèles et la bureaucratisation montrent que l'ADI a atteint une masse critique, tandis que les cadres estiment que les ressources classiques sont désormais insuffisantes pour maintenir la dynamique du mouvement. À l'initiative de l'ADN Côte-d'Ivoire, des structures nationales de microfinance dites "Ançar Finances » ont été mises en place: AFCI pour Ançar Finances Côte-d'Ivoire créé en 2007-2008 et AFM pour Ançar Finances Mali créé en 2010 ${ }^{31}$. Mêlant l'idéologie tiers-mondiste de la Grameen Bank, l'altermondialisme et l'action caritative islamique, ces établissements offrent ici des microcrédits à un taux préférentiel conforme aux principes de la charîa, destinés aux membres et sections de l'association.

51 Mais les ambitions de l'ADI ne s'arrêtent pas là, car Ançar Finances est en réalité conçu comme la préfiguration d'une banque islamique, considérée comme étant seule susceptible de permettre le développement du mouvement : constructions immobilières à Banconi et dans les territoires; densification de son réseau d'écoles et de lycées, incluant la réalisation d'une université islamique; pérennisation et professionnalisation du mouvement à travers le salariat; et enfin accroissement des capacités de recrutement social. De l'aveu même des cadres, le système des cotisations et des dons a atteint ses limites, tandis que l'objectif est désormais d'acquérir une autonomie financière en accédant aux marchés financiers internationaux, où les taux de crédits sont évidemment bien meilleurs que dans les banques.

Si les projets des réformes financières en cours montrent que l'ADI se perçoit bel et bien comme une organisation soucieuse de son autonomie économique, cela ne doit pas faire perdre de vue que le principal producteur de richesses est Haïdara et ses prêcheurs, à la fois atout et faiblesse de l'ADI dans son effort de bureaucratisation. De ce point de vue, audelà de l'autonomie financière, créer une banque islamique permettrait à l'ADI d'échapper, ou au moins de pondérer la logique charismatique qui assure l'essentiel de ses revenus, Haïdara et ses prêcheurs témoignant là d'un entreprenariat religieux solidement éprouvé. 


\section{Haïdara, ou la maîtrise d'un management religieux axé sur le peuple}

Au-delà de la personnalité de Haïdara, de sa capacité à se renouveler dans une sorte de continuité apparente, de sa volonté de réformer les pratiques de l'islam et de l'institutionnalisation de son mouvement, le guide de Ançar Dine a su requérir et adapter des techniques de prosélytisme qui lui ont permis de capitaliser son parcours religieux, au sens trivial du terme. À cet égard, on peut parler ici d'un véritable travail de management fondé sur une approche populaire qui se décline en trois stratégies convergentes: celle des langues nationales; celle de la médiatisation; et celle de l'événement, au terme desquelles il va alors mettre en place la pratique, la dimension concrète de son réformisme qu'est la bay'a.

\section{Prêcher malien : la stratégie des langues nationales}

C'est la technique du prêche et surtout son traitement en termes de diffusion qui va singulariser la démarche de Haïdara, et ce dès le milieu des années 1970. Dorothea Schulz (2003) a bien analysé la technique médiatique des sermons de Haïdara qui privilégie les langues vernaculaires. Il n'a pourtant pas été le premier à y recourir, et une longue tradition du prêcheur itinérant a marqué l'espace sahélien. De plus, Haïdara s'est fortement inspiré d'autres prêcheurs maliens, notamment Karamoko Lassana à Mopti et Abdoul Aziz Djiré à Ségou et Bamako. Mais Haïdara se singularise ici par le choix revendiqué de prêcher au moyen de ce que l'on appelle les «langues nationales », une approche justifiée dans sa biographie officielle, où il revendique un islam intégré à la culture ouest-africaine en posant la question suivante: "Nous apprenons le sens du Coran dans d'autres langues. Mais pourquoi partageons-nous le fait de ne pas prier Allah dans d'autres langues? » (Traoré $2007: 16)$.

Nous ne rentrerons pas ici dans la polémique que Haïdara avait suscitée en suggérant de pouvoir prier en langue nationale, contestant ainsi la sacralité de l'arabe liturgique, polémique qu'il bottera d'ailleurs en touche en déclarant qu'il avait été mal compris. Nous n'insisterons pas non plus sur le fait que le biographe est un militant du mouvement nko et qu'il défend là un point majeur du « prophétisme scripturaire » du nko (Amselle 2001 : 112) autour de l'africanisation de la lecture du monde. Cette perspective, Haïdara et l'ADI l'ont faite leur, si l'on en juge à la banderole qui a accueilli les fidèles lors du Maouloud 2011 et disant en langue nko : « Le maître souhaite la bienvenue à tous ceux qui viennent. Tous les Ançars doivent s'efforcer d'apprendre le Nko ${ }^{32}$. S'il est vrai que le nko n'est pas sans lien avec l'idéal fédéral du mouvement, Haïdara accorde toutefois une attention particulière aux langues vernaculaires - ce que n'est pas le nko -, d'abord parce qu'elles portent de l'identité, ensuite parce qu'elles constituent le média le plus approprié à l'égard d'un public essentiellement illettré. Enfin, si Haïdara requiert les langues nationales, c'est parce qu'il considère que les musulmans maliens doivent comprendre leur religion. Pour sa part, il prêche en bamanan kan, sa langue maternelle, qui constitue la langue véhiculaire au Mali et dans une partie de la sous-région à travers ses variantes.

Si l'homme maitrise tous les ressorts de cette langue, s'il est célèbre pour sa voix de stentor, s'il est réputé être aussi un rhétoricien incomparable mêlant autorité (barika), clarté (kènè kan) et humour (yèlèko), et s'il joue de la représentation populaire selon 
laquelle les Bamananw disent la "vérité nette » ("tiyèn jalan »), ses prêches invitent en fait chaque fidèle à considérer l'islam comme une religion universelle dont il participe en propre, et non une religion centrée sur le monde arabe tel que le suggère la réforme sunnite et son option salafite. Les prêches de Haïdara offrent ainsi aux fidèles le sentiment de saisir la complexité du monde dans leur langue et d'accéder alors à une intelligibilité du religieux en activant leurs propres codes culturels, notamment le style narratif griotique et l'imaginaire ségovien - ce dernier étant aussi paradoxalement "païen ", en référence à la bamananya (Colleyn 2004) - faisant cette fois-ci rupture non plus seulement avec l'arabe, mais encore avec la francophonie des élites administratives et politiques.

Mais ce qui aura distingué Haïdara des autres prêcheurs maliens utilisant eux aussi les langues nationales, c'est sa stratégie de diffusion de ses prêches. Car s'il est un " entrepreneur religieux », il est avant tout un infatigable "travailleur religieux » qui aura développé une intense activité tout au long de ses trente-cinq ans de carrière. Nous avons ainsi collecté, de façon non exhaustive, les cassettes audio de 280 prêches effectués par Haïdara au Mali et en Côte-d'Ivoire de 1982 à 2011, soit une moyenne de neuf à dix sermons par an, en dépit des deux suspensions. Certes, un imam prêche chaque vendredi dans sa mosquée, mais le prosélytisme de Haïdara est celui d'un islam hors mosquée et itinérant qui s'adresse aux communautés non localisées ou non établies socialement. De plus, et parce qu'ils ne se situent pas dans une logique nationale et internationale, les prêches de Haïdara sont d'une autre teneur que les sermons du vendredi, en portant toujours une dimension politique et de critique sociale qu'aucune mosquée ne saurait assumer sans risque.

\section{Diffuser le prêche : la stratégie de la médiatisation populaire}

Haïdara a longtemps été dans l'impossibilité d'accéder aux médias audiovisuels, qu'il s'agisse de la radio nationale, des radios FM ou de la télévision. Avant 1991, le pouvoir s'opposait à ce qu'il puisse intervenir sur la Radio nationale, y compris dans les émissions confessionnelles contrôlées alors par l'AMUPI. Mais en dépit de l'apparition des radios associatives et commerciales à partir de 1991, le régime démocratique de Alpha Oumar Konaré continuait à surveiller de près Haïdara. La première émission radiophonique à laquelle il participa date du 1er juillet 1994. Elle se déroula à la station de Koutiala de la radio associative Radio Kayira, avec l'accord du directeur du réseau national Oumar Mariko, actuel député du parti SADI. Si les autorités désapprouvèrent l'initiative et prirent même des mesures de rétorsion à l'encontre de la station, à partir de 1996, elles laissent désormais Haïdara intervenir sur les ondes, notamment sur Radio Djèkafo, Radio Patriote, Radio Benkan, Radio Kayira, Radio Liberté et même Radio Bamakan, la première radio libre fondée par Alpha Oumar Konaré en 1991.

Depuis 2008, Ançar Dine dispose de sa propre radio FM, qui émet sur la fréquence $92.9 \mathrm{mhz}$. Située dans le quartier périphérique de Kalaban Koro, à Bamako, elle porte le nom de La Voix du Citoyen, mais les Bamakois l'appellent simplement Radio Citoyen. L'essentiel de son contenu est religieux, mais elle se veut néanmoins une radio " commerciale", avec sponsors et contrats publicitaires, alternant programmes de variétés et programmes religieux ouverts dans la rubrique " espace partenaires » à tout prêcheur, moyennant finance. Si les interventions de Haïdara sur les radios FM sont en langue nationale, Ançar Dine dispose aussi d'un site Internet francophone, par lequel 
Radio Citoyen est diffusée sur l'Internet, tandis qu'une page Facebook en français ${ }^{33}$ lui a été dédiée en 2010.

Cette stratégie de médiatisation populaire par le biais des radios FM ou de l'Internet, en bamanan kan lorsque la communication est orale et en français lorsqu'elle est écrite, s'élargit désormais avec un nouveau projet visant à obtenir une licence de diffusion d'une chaîne de télévision qui permettrait à Ançar Dine de «servir davantage les fidèles musulmans $»^{34}$. Mais bien avant de pouvoir accéder aux radios FM, de créer la sienne et de bénéficier d'une éventuelle chaîne de télévision, Haïdara avait opté très tôt dans sa carrière pour le principe d'une médiatisation populaire, apparaissant de ce point de vue comme un précurseur au Mali. C'est en effet en enregistrant ses sermons sur des supports bon marché, cassettes audio analogiques et plus récemment vidéos- $\mathrm{CD}$, qu'il a innové et fait rupture avec le prêche itinérant traditionnel, accédant ainsi à une dimension nouvelle du prosélytisme islamique malien (Schulz 2003, 2007).

Cette aventure médiatique débute en 1977. Haïdara est alors en Côte-d'Ivoire où il enregistre probablement sa première cassette analogique, dont nous avons pu nous procurer une copie. Contrairement à la plupart des cassettes enregistrées à partir de 1982 à l'occasion de cérémonies religieuses, de débats ou à la radio, cette première cassette a la particularité d'avoir été réalisée en "mode studio", c'est-à-dire sans public, en mobilisant juste les trois protagonistes que sont le prêcheur (wajulikèla) Haïdara, un « lecteur coranique » (" kuranè kolaminèna ») et un « agent rythmique » (" naaminèna »).

Haïdara ne fait aucun commentaire sur ce prototype, ni d'ailleurs sur les contraintes techniques et financières auxquelles il a dû être confronté pour la duplication de la bande originale. En réalité, et en dépit de l'impact populaire que ces cassettes lui auront offert, Haïdara ne parle guère de ses enregistrements passés. Depuis qu'il est devenu une des figures majeures de l'islam malien, envers qui le respect est tel qu'on le fait parler après tout le monde de manière à ce qu'il ne puisse être contredit lors des rencontres publiques entre religieux, Haïdara a demandé de ne plus diffuser ses anciennes cassettes aux propos souvent virulents et parfois contestables, y compris vis-à-vis du positionnement actuel de Ançar Dine.

63 En revanche, il explique volontiers comment il découvrit, à la fin des années 1970, la cassette d'un prêcheur égyptien, le cheikh salafite 'Abd al-Hamîd Kishk ${ }^{35}$, qui circulait en Côte-d'Ivoire et qui va littéralement bouleverser sa carrière en devenir. Mais ce n'est qu'en 1985 (ou peut-être en 1987, l'année où il se rend pour la première fois à La Mecque), que Haïdara rencontra Kishk, un personnage charismatique qui drainait de véritables foules lors de ses prêches du vendredi au Caire (Kepel 1984) et dont les sermons enregistrés sur cassettes, diffusés dès le début des années 1970, vont atteindre les musulmans arabophones de tous les continents (Eickelman 1999 : 36).

Haïdara s'est fortement inspiré de Kishk du point de vue de la scénographie et des effets de ses prêches, s'efforçant de reprendre la technique de l'humour populaire dont Kishk usait et qui rendait ses sermons, parfois transgressifs, souvent divertissants et interactifs (Kreil 2010). Mais l'influence de Kishk tient surtout à cette technique d'enregistrement sur cassettes que Haïdara va reprendre bien avant leur rencontre au Caire. Si certains estiment que la faconde de Haïdara tient beaucoup de sa rencontre avec le prêcheur Abdoul Aziz Djiré dont on a déjà parlé, l'influence de Kishk est indiscutable; Haïdara appellera d'ailleurs le premier fils qu'il a eu de sa seconde épouse Hadjaratou Tounkara, 
au début des années 2000, Abdoul Hamid Keshky ${ }^{36}$ en témoignage de son admiration pour le tribun égyptien.

Toutefois, la rentabilité de ce qu'il convient d'appeler la « méthode kishk » et l'usage des small medias (Sreberny-Mohammadi \& Mohammadi 1994) dans la stratégie de médiatisation populaire de Haïdara vont s'imposer définitivement en 1988. C'est à cette date qu'il enregistrera, sans doute pour la première fois, son prêche du Maouloud, témoignant ainsi de son souhait de transformer cette fête religieuse plutôt discrète à Bamako, mais dont il a déjà pu tester le caractère de spectacle populaire dès 1984, en un événement public dédié à son prosélytisme.

\section{Diffuser le prêche : la stratégie de l'événement}

Si l'islam prêché par Haïdara n'est pas un islam de mosquée, il est en revanche un islam de place publique qui s'inscrit dans une tradition de la prédication spectaculaire et populaire. Aussi, ce type de prêches requiert-il un caractère événementiel préalable qui justifie une telle prise de parole en public: Ramadan ou Tabaski. Mais la mosquée se révèle concurrente sur ces deux fêtes, et c'est la raison pour laquelle Haïdara va investir un événement très peu pris en charge par les mosquées, bien que prisé par les confréries centrées sur des zawiya, comme la Tijâniyya Hamawiyya de Nioro-du-Sahel ou la Qadiriyya de Dilly: le Maouloud, selon l'orthographe utilisée au Mali, qui marque l'anniversaire de la naissance du Prophète.

67 Nous avons développé ailleurs les enjeux locaux de cette cérémonie (Holder 2009) qui présente la singularité de n'avoir aucun caractère obligatoire. Jusqu'au milieu des années 1990, à l'exception des régions Nord et Est du Mali, le Maouloud était d'ailleurs une célébration peu visible effectuée par certaines familles pieuses. Du reste, même à Tombouctou et à Djenné, où il était fêté de longue date avec des louanges récitées nuitamment en l'honneur du Prophète, le Maouloud était prétexte à des manifestations que d'aucuns qualifient de carnavalesques - voire de bid'a pour les sunnites réformés -, transgressant rituellement les normes sociales, les rapports de genre et les relations de pouvoir.

Mais Haïdara va transformer, depuis Bamako, cette cérémonie marquée par une forte identité du Nord et de l'Est, en un rendez-vous annuel majeur de la piété populaire et de l'identité nationale dédiées à son propre mouvement. Dès le début des années 1990, l'organisation matérielle et la scénographie du prêche du Maouloud conçues par Ançar Dine sont fixées : sous l'éclairage de néons électriques, un alignement de tables derrière lesquelles se répartissent les membres du bureau ou de la section locale; en arrière-fond, une large banderole du mouvement Ançar Dine à l'effigie de Haïdara; aux quatre coins d'un grand espace rectangulaire, des pieux sur lesquels sont attachés néons et hautparleurs; au centre de l'alignement des tables, le prêcheur avec un micro ; à sa droite, le lecteur coranique qui cite les sourates que le prêcheur commente en langue vernaculaire à partir des hadîth; face au prêcheur et dos au public, l'agent rythmique qui ponctue chaque phrase par un naam emphatique.

Les cérémonies commencent généralement vers vingt heures par l'écoute préalable de louanges au Prophète (zikiriw, de l'arabe dhikr) et en l'honneur du guide de Ançar Dine, chantées en direct ou, plus souvent, retransmises par enregistrements. Puis un ou plusieurs prêcheurs, assistés de louangeurs (zikiridalaw) qui se disent "griots de Dieu » ("ala jeliw»), commencent à chauffer le public en attendant l'arrivée du prêcheur 
principal. S'agissant du Maouloud animé par Haïdara, l'arrivée vers vingt-deux heures de ce dernier, entouré d'une imposante escorte du service de sécurité interne et d'agents de la force publique, provoque un mouvement de foule indescriptible: des femmes s'évanouissent et sont vivement évacuées, tandis que la foule crie la devise religieuse de Haïdara : «yaa bani!» Le tribun va alors tenir en haleine un public surtout constitué de femmes, de jeunes et de gens de condition modeste pendant deux ou trois heures de sermons où alternent crainte du jugement de l'au-delà, critiques sociales à l'adresse du pouvoir, recommandations sur le mariage, anecdotes chrétiennes ou "païennes" mettant en perspective les pratiques musulmanes, traits d'humour et ironie. De son côté, le public lance des "Allah Akbar» qui ponctuent les montées dramatiques du prêche, tandis qu'au détour d'un long commentaire, les rires fusent et, parfois, de timides applaudissements se font entendre.

70 À mesure que le Maouloud de Haïdara se popularise, il va occuper une position centrale dans l'organisation de Ançar Dine, à commencer par l'économie financière. C'est à cette occasion que seront réunis les cotisations et dons, présentés comme une sorte de zakât alFitr, l'aumône rituelle que les mosquées sont normalement seules habilitées à recueillir au Ramadan. Mais le Maouloud va devenir tout aussi central du point de vue de l'économie politique de Ançar Dine, jusqu'à lui donner en 2001 le statut de congrès, puis de conférence internationale en 2008. On profite ainsi de cet événement mobilisateur pour effectuer le bilan moral et financier de l'ADI, renouveler ses instances et fixer les objectifs de l'année à venir. Enfin, le Maouloud organisé par Ançar Dine va parallèlement être conçu comme une sorte de plateforme nationale du prosélytisme, où l'enjeu est de « fidéliser » les publics, aux sens à la fois propre et commun du terme.

71 Constitué en un lieu de publicisation du réformisme populaire porté par Haïdara, le Maouloud attire une foule de plus en plus nombreuse d'année en année, venue écouter les enseignements du maître et sa dénonciation des injustices du monde. De 1984 à la fin des années 1990, Haïdara prêche sur la place de Railda déjà mentionnée, jusqu'à ce qu'elle fasse l'objet d'une série d'aménagements urbains. Arguant du fait qu'il mobilisait des milliers de fidèles, Haïdara obtient alors des autorités, au tournant des années 2000, l'autorisation d'organiser son Maouloud sur le champ de course de l'hippodrome, avant de s'installer au stade omnisport Modibo Kéita de Bamako en 2003 (Soares 2005 : 254). C'est là qu'il officie toujours, réunissant plus de 35000 personnes ${ }^{37}$ au fil de prêches en stade qui ne sont pas sans évoquer ceux des télévangélistes.

72 Si le choix de ce stade est consécutif de l'importance du public et du fait que Bamako, soumise à une urbanisation sans précédent, a fort peu d'espaces appropriés, il n'est toutefois pas anodin que ce Maouloud se déroule sur un stade baptisé du nom de Modibo Kéita. De fait, Haïdara célèbre son Maouloud en bénéficiant de la symbolique particulière de l'indépendance nationale et de son héros Modibo Kéita, dont les échos se mesurent aux sons du chant Mali entonné à l'ouverture de chaque prêche par la foule des fidèles. Composé en 1960 par Djéli Bazoumana Cissoko à la gloire de la nouvelle République indépendante, le chant a en effet été adapté par un louangeur Ançar pour devenir l'hymne de l'ADN du Mali, à l'instar du chant Folikan considéré comme l'hymne de l'ADI.

73 En moins de trente ans, Haïdara et Ançar Dine auront ainsi fait du Maouloud le lieu central de la doctrine identitaire et sociale de ce mouvement religieux en imposant leur "marque de fabrique », bientôt copiée et généralisée sur tout le territoire par les jeunes prêcheurs qui ne disposent pas de mosquée, mais aussi par les structures plus traditionnelles du monde soufi que sont les "villes bénies" et les confréries. Si le 
Maouloud était célébré avec plus ou moins d'éclat par les unes et les autres bien avant Haïdara, il va être massivement réinvesti et devenir, à la fin des années 1990, un nouvel enjeu de visibilité et de recrutement de la sphère islamique malékite, au grand dam des sunnites réformés.

Profitant de cet événement annuel qu'est devenu le Maouloud, Ançar Dine va mettre enfin en scène la prononciation collective de sa bay' $a$, conformément à l'un des objectifs de l'ADI, dont les statuts de 2008 stipulent d'« œuvrer au perfectionnement individuel et collectif des fidèles par la sensibilisation et l'appel à la prestation de la Baya " (art. 4). Présentée sous la forme d'un serment d'engagement effectué en public, la bay'a aurait ainsi été prononcée par plus de 2000 personnes au Maouloud 2011 de Bamako, lors de la nuit du second prêche dédié à la dation du nom du Prophète. Cette prononciation les aura fait devenir, non pas encore Ançars, mais des musulmans confirmés. En effet, si Ançar Dine est connu (et critiqué) pour sa pratique ostensible de la bay' $a$, le mouvement exige en réalité deux bay' $a$ distinctes qui réfèrent à un engagement en deux temps à la cause : la Bay'a al-Islamiyya et la Bay'a al-Rizwân.

\section{Une bay'a, deux bay'a : se réengager avant d'adhérer à Ançar Dine}

La Bay'a al-Islamiyya est la plus connue et souvent considérée comme la seule à être prononcée, tandis qu'elle fait aussi l'objet d'une critique sévère de la part de nombreux religieux. La Bay'a al-Islamiyya est un serment islamique conçu par Ançar Dine comme un « réengagement » individuel effectué par celui qui, né musulman, aspire à faire le choix conscient d'être musulman. Contestant ainsi implicitement l'autorité de la " profession de foi » ("shahâda »), considérée par Haïdara comme relevant de la conversion, la Bay"a alIslamiyya est solennisée par six promesses contraignantes: $41^{\circ}$ ) Je n'associerai rien à Allah. $2^{\circ}$ ) Je ne volerai jamais. $3^{\circ}$ ) Je ne commettrai pas l'adultère. $4^{\circ}$ ) Je ne tuerai jamais mes enfants. $5^{\circ}$ ) Je ne commettrai aucune infamie ni sous les mains ni sous les pieds. $6^{\circ}$ ) Je ne désobéirai jamais au Prophète (SAS) en ce qui est convenable $»^{38}$.

Si Ançar Dine est fustigé par sa pratique de la bay'a, il s'agit en réalité d'un serment bien connu du monde soufi, y compris malien, par lequel le maitre et son mouride s'engagent réciproquement. La référence coranique de cette bay'a confrérique est le verset 10 de la sourate $48 \mathrm{Al}$-Fath, "La Victoire éclatante", qui renvoie au "Serment sous l'Arbre" ("Bay'a al-Shajarah ») effectué au lieu-dit Hudaybiyya et qui est connu aussi sous le nom de "Serment de la Satisfaction » ("Bay'a al-Rizwân»).

Pourtant, si la bay ${ }^{\prime} a$ a un fondement coranique et qu'elle est pratiquée dans les confréries soufies ouest-africaines, le serment de réengagement dit Bay'a al-Islamiyya qu'exige Ançar Dine est d'une autre nature, car il implique le fidèle, non pas vis-à-vis d'un maitre ou d'un guide, mais de l'islam en tant que religion. En ce sens, cette bay' $a$ de Ançar Dine n'est pas sans rappeler les fondements de la réforme sunnite, qui se singularise précisément par le fait qu'elle préconise une relation non médiatisée avec Dieu et son prophète. Aussi, en dépit de sa forte similitude, la Bay'a al-Islamiyya de Ançar Dine n'est nullement tirée du "Serment sous l'Arbre » de Hudaybiyya, mais du "Premier Serment d'Allégeance à Al-'Aqaba » dont nous avons déjà parlé plus haut. Cette source est livrée par le laudateur attitré de Haïdara, Nouhoum Dembélé, dans son adaptation du chant Folikan servant d'hymne à l'ADI et qui dit : «Il [Haïdara] a révélé le mot Bay‘a [du Sahîh] de al-Bukhârî. »

Sans entrer dans le détail des classifications des hadîth, dont l'enjeu est considérable du point de vue doctrinal, cette louange informe que la Bay'a al-Islamiyya a non seulement 
une base coranique, mais qu'elle est fondée sur les hadith « intégralement authentiques » ( "jâmi' us-sahîh ») reconnus par le monde sunnite. Il s'agit en l'occurrence du Sahîh de AlBukhârî, plus particulièrement du hadîth n 232 dit «Du serment de loyauté de "Aqaba ", extrait du Livre des mérites des Ansârs ${ }^{39}$. De nombreux autres hadîth évoquent le principe de la bay'a, mais c'est celle donnée à Al-'Aqaba qui fait ici référence et permet le réengagement éclairé du musulman dans l'islam, selon la doctrine de Ançar Dine. On notera ici le soin particulier dont Haïdara et Ançar Dine ont pu faire preuve pour identifier cette référence savante, veillant à se démarquer de la bay'a soufie classique qui se fonde sur le Coran. Mais cette Bay'a al-Islamiyya n'est pourtant qu'une étape avant que le fidèle n'adhère au mouvement. Après le « réengagement » dans l'islam, c'est en effet l'engagement dans Ançar Dine qui intervient, phase ultime de l'acquisition de l'identité ançar et qui est là encore solennisée par un serment appelé Bay $a$ al-Rizwân, lequel fait référence cette fois-ci au « Serment de l'Arbre » de Hudaybiyya.

Le rituel de la Bay'a al-Rizwân est assez similaire à celui de la Bay'a al-Islamiyya, mais le contenu est cette fois conforme à l'esprit du serment confrérique. Ainsi, lors de l'Assemblée internationale du Maouloud 2011 qui se tenait au sein de la mosquée du vendredi des Ançars encore en travaux, les membres du nouveau bureau de l'ADI ont chacun leur tour prêté serment à Haïdara, la main posée sur le Coran, en répétant la formule suivante : «Allah est mon témoin ; le Coran est mon témoin; le maître est mon témoin; vous les musulmans êtes mes témoins; si je trahis la confiance du maître, qu'Allah et le Coran abolissent mon avenir. »Ce serment d'engagement vis-à-vis de Ançar Dine, ici médiatisé par Haïdara (ou un prêcheur du whoudouh lorsque le rituel se déroule dans le cadre d'une section locale), réintroduit de façon centrale le pouvoir charismatique - d'autant que dans le cas d'espèce, ce sont les instances suprêmes de l'ADI qui prêtent serment à Haïdara - et révèle l'ampleur du problème auquel est confronté l'ADI comme organisation bureaucratique au service d'une cause religieuse. Sa légitimité dépend en effet du serment au guide spirituel ou à ses représentants, tout comme le recrutement de nouveaux membres.

Au-delà du jeu des références religieuses que suscite l'exercice de ces deux bay'a et du sentiment qu'elles donnent de l'extérieur de correspondre aux caractéristiques d'une secte, pris au sens de ce qui retranche de la communauté globale, on peut considérer son principe comme une technique de recrutement efficace vis-à-vis de ceux qui souhaitent éprouver les contraintes d'un engagement authentique. En réalité, la suspicion sectaire de ce mouvement autour de la pratique de la bay'a, pris comme serment de fidélité absolue et fanatique, se rapporte non pas au positionnement religieux - qui reste dans le sunnisme malékite -, ni au charisme de Haïdara - qui s'inscrit dans une longue tradition de l'islam sahélien -, mais à la logique d'autonomie que Ançar Dine affiche tous azimuts : autonomie d'une pensée sociale revendiquant une inquiétante "raison populiste» (Laclau 2008); autonomie organisationnelle et financière d'une association de la société civile internationaliste; autonomie du point de vue de la nature d'un mouvement, ni totalement confrérie ni strictement association, où l'engagement religieux confine à un engagement politique. Aussi, en mêlant de la sorte ces deux registres dont la fusion est considérée a priori contre-nature, certains estiment qu'il s'agit bel et bien d'un mouvement sectaire en rupture avec les normes de l'ordre global.

81

$\mathrm{Au}$ terme de cette étude, on peut sans doute se demander comment le prêcheur Chérif Ousmane Madani Haïdara est ainsi devenu cet acteur dominant de la scène religieuse 
malienne. Pour quelles raisons attire-t-il, non seulement des gens modestes et souvent analphabètes, des femmes et des jeunes sans qualification ou des paysans paupérisés, mais aussi des intellectuels, des acteurs politiques, des fonctionnaires et des entrepreneurs économiques? Si Haïdara et son prosélytisme sont loin de faire l'unanimité et si nombreux sont ceux qui pensent qu'il profite de sa position et de son autorité religieuse pour s'enrichir, il n'en demeure pas moins la figure du cadet social ayant conquis son autonomie seul et incarnant ainsi le modèle valorisé de l'« entrepreneur » («nyangara »). Haïdara est un héros populaire qui porte en lui un destin et l'accomplit en dépit des difficultés, un idéal de réalisation personnelle auquel chacun peut se référer lorsque l'ordre social est devenu si fermé. Certes, il n'est pas le seul modèle possible, mais il incarne une autorité acquise au Mali en requérant la voie de la légitimité la plus forte qu'est l'islam. Ce faisant, il redéfinit les valeurs d'autonomie, de réussite et de changement au prisme d'un engagement religieux qui se conscientise et s'organise à travers le mouvement Ançar Dine.

Mais si chacun peut avoir une idée de qui est ou n'est pas Haïdara, peu nombreux sont ceux qui savent finalement ce qu'est Ançar Dine et vers quoi tend ce mouvement. Nous avons proposé à cet égard l'hypothèse de l'autonomie, et d'abord celle qu'incarne le guide lui-même, qui a conçu avant tout ce mouvement comme le moyen d'y parvenir. Toutefois, en ayant pu rassembler ainsi 70000 fidèles autour de lui, Haïdara se voit désormais confronté à une tension structurelle qui oscille entre un réformisme qu'il situe hors des voies soufies et une représentation populaire de la guidance qui demeure celle du cheikh. C'est en partie dans la résolution de ce dilemme que prend sens tout l'effort conduit par le prêcheur à se vouloir un « chérif ».

$\mathrm{Si}$, comme le soulignent certains hadîth, le cheikh parmi ses disciples est comme le Prophète dans sa communauté, pour un chérif, il est donc possible de se passer du statut de cheikh en se reliant directement au prophète. Aussi, non seulement la bay'a que le fidèle ançar fait à Haïdara équivaut à l'engagement entre le murîd et son cheikh, mais parce qu'il n'est aucunement dans une voie spécifique, Haïdara fait en quelque sorte figure de " cheikh achevé ", un modèle de guidance autonome qu'a pu revendiquer en son temps Al-Hajj 'Umar al-Fûtî, dont le djihad s'est arrêté à Ségou (Robinson 1988) et dont l'idéal religieux renaîtrait à travers Haïdara le ségovien. Parce qu'il se proclame descendant des chérifs de La Mecque, Haïdara n'aurait lui aussi aucune obligation à se référer à une voie. « Le Shaykh véritable [...] est l'héritier du prophète, qui guide et appelle vers le droit chemin », écrivait Al-Hajj 'Umar. «Pour avoir franchi toutes les étapes de l'initiation sous la direction d'un autre Shaykh-héritier (un wârith) dont la chaîne de garants remonte jusqu'au prophète, le Shaykh édificateur reçoit les lumières divines et les insu[f]fle à ses disciples. Un tel Shaykh n'est pas tenu de se lier à une école juridique. Il a un accès direct à la vérité. Il est, pour ses disciples, une porte ouverte vers Dieu $»^{40}$. On ne saurait identifier le «Shaykh-héritier » qui initia Haïdara, sinon peut-être le Prophète luimême, comme tend à le suggérer finalement le réformisme ançar. Et à l'instar de Al-Hajj 'Umar, chérif Haïdara est considéré par ses fidèles, et même au-delà, comme un " pôle " ( " kutubu », de l'arabe qutub) qui, à ce titre, en fait l'intermédiaire direct avec le Prophète.

De toute évidence, on voit mal comment une telle guidance pourrait échoir à un successeur qui, selon les statuts de l'ADI, « est toujours désigné par la famille et parmi la descendance du cheikh Chérif Ousmane Madani Haïdara " (art. 12). Cette autonomie charismatique, qui aura ostracisé Haïdara vis-à-vis de la sphère islamique avant qu'il ne finisse par la subordonner, pose problème au mouvement Ançar Dine lui-même. Si 
l'institutionnalisation qui a accompagné la création de l'Association malienne pour le soutien de l'islam était attendue, la bureaucratisation de Ançar Dine et sa transformation en ADI ont conduit le mouvement vers une logique d'autonomie quasi incompatible qui est celle d'une figure de l'État hors de l'État. Le modèle étatique est certes une référence prégnante au Mali, et Ançar Dine ne manque pas d'y puiser pour développer ce qui est bel et bien un projet d'autonomie. Mais si la figure de l'État se fonde sur une tension permanente entre pouvoir bureaucratique et pouvoir charismatique, la ritualisation de ce dernier est au cœur de la transformation d'une organisation de la cité (idéale ou pas) en un État. Et c'est sans doute dans ce sens qu'il faut comprendre les tentatives que déploie désormais l'ADI pour contrôler les prêcheurs du mouvement.

Répondant récemment à JournalduMali.com à la question: «La gestion du bien public rime-t-elle avec islam?", Haïdara explique : "Si la politique veut dire promouvoir la bonne gestion du bien public", alors lui Haïdara fait de la politique. Et serait prêt à faire allégeance avec l'homme politique qui incarnera le plus les valeurs qu'il défend. Cependant, a-t-il précisé, Haïdara n'en a cure de devenir président de la République du Mali » (Fofana 2010). Cette reconnaissance de la singularité de la sphère étatique est-elle l'opinion partagée par les membres dirigeants de Ançar Dine ? Pour eux, le devenir de l' ADI est-il de servir la guidance de Haïdara et ses héritiers, de fonder une nouvelle " voie " ( "tarîqa») qui demeurerait distincte du champ politique, ou de se penser au contraire comme une organisation autonome porteuse d'un projet étatique alternatif? Et surtout, de quel côté pèse finalement le peuple des Ançars : vers son guide spirituel ou vers cette organisation fédérale qu'est l'ADI ? L'aventure non achevée de Ançar Dine international est-elle celle des Ançars de la République ou d'une République des Ançars?

\section{BIBLIOGRAPHIE}

AMSELLE, J.-L.

1985 - « Le Wahabisme à Bamako (1945-1985) », Canadian Journal of African Studies, XIX (2) :

345-357.

2001 - Branchements. Anthropologie de l'universalité des cultures, Paris, Flammarion.

ANONYME

2009 - « La division gagne le rang des associations musulmanes : Chérif Ousmane Madani Haïdara attaque Mahmoud Dicko ", Bamako, L'Indicateur du Renouveau, 25 septembre.

BARRY, $\mathrm{H}$.

2004 - Les charmes discrets de Bamako, Bamako, ADC/ML-Djénè-Color.

BAXTER, J.

2002 - « Mali’s Muslim Majority Enters Political Fray », BBC-News World-Africa, 22 avril.

BOURDARIAS, F.

2008 - « L'imam, le soufi et Satan : religion et politique à Bamako (Mali)», in F. BOURDARIAS \& H. BERTHELEU (dir.), Les constructions locales du politique, Tours, Presses de l'Université François

Rabelais : 115-139. 
BRENNER, L.

$1991 \mathrm{a}$ - « Introduction : essai socio-historique sur l'enseignement islamique au Mali », in B. SANANKOUA \& L. BRENNER (dir.), L'enseignement islamique au Mali, Bamako, Jamana : 1-23.

1991 b - « Médersas au Mali. Transformation d'une institution islamique », in B. SANANKOUA \& L. BRENNER (dir.), op. cit. : 63-85.

COLLEYN, J.-P.

2004 - « L'alliance, le dieu, l'objet », L'Homme, 170 : 61-78.

COULIBALY, O.

2011 - « Bani, préoccupé par l'union des musulmans du Mali... », Le Challenger, er $^{\mathrm{er}}$ mars.

DAVIS, K.

2002 - Preaching to the Converted. Charismatic Leadership, Performances and Electronic Media in Contemporary Islamic Communities, Mémoire de Master, Montréal, Université Concordia.

EICKELMAN, D. F.

1999 - « Communication and Control in the Middle East : Publication and its Discontents », in D. F. EICKELMAN \& J. W. ANDERSON (eds.), New Media in the Muslim World: The Emerging Public Sphere, Bloomington-Indianapolis, Indiana University Press : 33-43.

FOFANA, M.

2010 - « Chérif Ousmane Haïdara : prêche et télévision font-ils bon ménage ? », $<$ JournalduMali.com>, 10 mars.

GORDON, J.

2004 - « Abd al-hamid kishk », in R. C. MARTIN (ed.), Encyclopedia of Islam and the Muslim World, Oxford, Macmillan : 2-3.

GUEYE, C.

2002 - Touba. La capitale des Mourides, Paris, Karthala.

HAMÈs, C.

1980 - « Deux aspects du fondamentalisme islamique », Archives de sciences sociales des religions, 50 (2) : 177-190.

HOLDER, G.

2009 - «"Maouloud 2006”, de Bamako à Tombouctou. Entre réislamisation de la nation et laïcité de l'État : la construction d'un espace public religieux au Mali », in G. HOLDER (dir.), L'islam, nouvel espace public en Afrique, Paris, Karthala (« Les Terrains du Siècle ») : 237-289.

2012 - « Djenné, “la ville aux 313 saints”. Convocation des savoirs, "lutte des classements” et production d'une ville sainte au Mali », Cahiers d'Études africaines [sous presse].

KEPEL, G.

1984 - Le Prophète et le pharaon, Paris, La Découverte.

KREIL, A.

2010 - « Se faire cheikh au Caire », Archives de sciences sociales des religions, 149 (1) : 255-272.

LACLAU, E.

2008 - La raison populiste, Paris, Éditions du Seuil [1 $1^{\text {re }}$ édition américaine, Verso 2005].

LY-TALL, M.

1991 - Un islam militant en Afrique de l'Ouest au XIX ${ }^{e}$ siècle : la Tijaniyya de Saiku Umar Futiyu contre les pouvoirs traditionnels et la puissance coloniale, Paris, L'Harmattan.

MAÏGA, K. B. A. 
s.d. - Cheick Soufi Bilal. Un pacifique conquérant, Plateau Anwar et CMS-Média, [vraisemblablement paru en mars-avril 2009].

MANLEY, A.

1997 - « The Sosso and the Haïdara : Two Muslim Lineages in Soudan français (1890-1960) », in D. ROBINSON \& J.-L. TRIAUD (dir.), Le temps des marabouts. Itinéraires et stratégies islamiques en Afrique occidentale française (1880-1960), Paris, Karthala : 319-336.

MARTY, P.

1920a - Études sur l'islam et les tribus du Soudan, t. I, Les Kounta de l'Est, les Berabich, les Iguellad, Paris, Ernest Leroux.

1920b - Études sur l'islam et les tribus du Soudan, t. IV, La région de Kayes-Le pays bambara-Le Sahel de Nioro, Paris, Ernest Leroux.

MAZZOLENI, F.

2008 - L'épopée de la musique africaine. Rythmes d'Afrique atlantique, Paris, Éditions Hors Collection. MEUNIER, M.

2010 - « Mali. L'imam qui casse le code », Jeune Afrique, 10-16 octobre.

NIEZEN, R. W.

1990 - « The "Community of Helpers of the Sunna" : Islamic Reform among the Songhay of Gao

(Mali)», Africa (London), 60 (3) : 399-424.

REICHMUTH, S.

1996 - «Education and the Growth of Religious Associations among Yoruba Muslims. The AnsarUd-Deen Society of Nigeria », Journal of Religion in Africa, 26 (4) : 365-405.

RHANI, Z.

2009 - « Le chérif et la possédée. Sainteté, rituel et pouvoir au Maroc », L’Homme, 190 : 27-50.

ROBINSON, D.

1988 - La guerre sainte d'al-Hajj Umar. Le Soudan occidental au milieu du XIX ${ }^{e}$ siècle, Paris, Karthala.

ROY, O.

1999 - « Le post-islamisme », Revue du monde musulman et de la Méditerranée, 85-86 : 11-30.

2001 - « Islam et politique en Asie centrale », Archives de sciences sociales des religions, 115 : 49-61.

SANANKOUA, B.

1991 - « L'enseignement islamique à la radio et à la télévision au Mali », in B. SANANKOUA \& L.

BRENNER (dir.), op. cit. : 127-141.

SCHULZ, D. E.

2003 - " "Charisma and Brotherhood" Revisited : Mass-mediated Forms of Spirituality in Urban

Mali », Journal of Religion in Africa, 33 (2) : 146-171.

2007 - « Evoking Moral Community, Fragmenting Muslim Discourse : Sermon Audi-recordings and the Reconfiguration of Public Debate in Mali », Journal for Islamic Studies, 27 : 39-72.

SOARES, B. F.

2005 - Islam and the Prayer Economy. History and Authority in a Malian Town, Edinburgh, Edinburgh University Press.

2006 - « Islam in Mali in the Neoliberal Era », African Affairs, 105 (418) : 77-95.

2007 - « Saint and Sufi in Contemporary Mali », in M. VAN BRUINESSEN \& J. D. HOWELL (eds.), Sufism and the « Modern » in Islam, London, IB Tauris : 76-91.

2009 - « The Attempt to Reform Family Law in Mali », Die Welt des Islams, 49 : 398-428.

2010 - «"Rasta" Sufis and Muslim Youth Culture in Mali », in L. HERRERA \& A. BAYAT (eds.), Being 
Young and Muslim. New Cultural Politics in the Global South and North, Oxford, Oxford University

Press : 241-257.

SREBERNY-MOHAMMADI, A. \& MOHAMMADI, A.

1994 - Small Media, Big Revolution : Communication, Culture and the Iranian Revolution, Minneapolis, University of Minnesota Press.

THIRIOT, C.

2010 - « Islam et espace public au Mali : une "société civile religieuse" très engagée ", in P.

SADRAN, R. OTAYEK \& D. DARBON (dir.), Altérité et identité, itinéraires croisés. Mélanges offerts à Christian

Coulon, Bruxelles, Bruylant : 213-245.

TRAORÉ, O. S., SANKARÉ, M. \& TOURÉ, A.

2007 - Qui est Haïdara ?, Bamako, Mahamoud Sankaré, 14 mars [fascicule en langue nko].

TRIAUD, J.-L.

1985 - « Les agents religieux islamiques en Afrique tropicale : réflexions autour d'un thème ", Canadian Journal of African Studies, 19 (2) : 271-282.

1986 - « Abd al-Rahman l'Africain (1908-1957), pionnier et précurseur du Wahhabisme au Mali », in O. CARRÉ \& P. DUMONT (dir.), Radicalismes islamiques, vol. 2, Paris, L’Harmattan : 162-179.

\section{NOTES}

1. Plutôt que de "wahhabisme ", on préférera parler ici de "sunnisme réformé ", en référence aux diverses tendances qui se réclament de la réforme néo-hanbalite engagée par Muhammad ibn Abd al-Wahhâb en Arabie au XVIII siècle et qui fait rupture avec le malékisme dans lequel se reconnaissent la plupart des musulmans en Afrique de l'Ouest.

2. Bamako comptait plus de 200 mosquées en 1988, contre 77 vingt ans plus tôt et 41 en 1960 (SANANKoua 1991: 127), mais certains avancent désormais le chiffre de 1000 mosquées (BARRY 2004 : 27).

3. Essentiellement suscités dans les radios commerciales et rediffusés sur des supports bon marchés (cassettes audio, vidéos- $\mathrm{CD}$, etc.), les débats charrient tous les problèmes de société : "insécurité routière", excision, cinquantenaire de l'indépendance, statut juridique de la femme, peine de mort, éducation et emploi des jeunes, émigration, etc. (H OLDER 2009 ; THIRIOT 2010).

4. Ainsi, l'imam Mahmoud Dicko aurait été successivement ou conjointement à la tête d'une vingtaine d'associations islamiques; voir BAXTER (2002).

5. Données tirées du nouveau site Internet de Ançar Dine : <http://ousmanehaidara.com>, consulté le 4 octobre 2010, qui a remplacé un premier site aujourd'hui fermé : <http:// www.ancardine-haidara.com>, consulté en juin 2006.

6. Les termes vernaculaires qui figurent dans ce texte sont exprimés en langue bamanan et sont transcrits en alphabet phonétique simplifié : $e=$ é; $\grave{e}=$ è ou $\hat{e} ; \grave{o}=0$ ouvert comme dans « hotte »; $c$ = la palatale occlusive sourde comme dans « check-list »; ny = la palatale nasale comme dans « nier »; $n g$ = la vélaire nasale comme dans « camping ».

7. Les données biographiques sur Haïdara sont issues d'un travail de synthèse à partir de trois sources croisées: 1 . un entretien en langue bamanan réalisé le 9 juillet 2008 au domicile de l'intéressé, à Bamako; 2. une interview recueillie par Kassim Traoré, titrée «Le prêcheur Séid Chérif Ousmane Madani : “La légitime défense n'est pas interdite en 
islam" ", qui est parue dans le journal Bamako Hebdo du 13 mars 2010 ; 3. un fascicule en langue nko intitulé Qui est Haïdara?, biographie officielle parue en 2007 et rédigée par trois fidèles de Haïdara, Oumar Traoré, l'auteur principal, assisté de Mahamoudou Sankaré et Aboubakar Touré. Nous devons à Soumaillla Camara, titulaire d'une maîtrise en anthropologie de l'Université de Bamako, le déchiffrage de ce fascicule.

8. Fils du ségovien Mamadi Kané, qui s'installa à Koulikoro vers 1900 (MARTY 1920b : 61), Lassana Kané dit Karamogo Lassana était réputé pour sa connaissance des hadith et fut l'un des grands prêcheurs tijânî dès la fin des années 1950. Ardent militant de l'US-RDA et prêchant en faveur de Modibo Kéïta, il fit sa carrière essentiellement à Mopti et Kayes et décéda vers 1981 ou 1982. L'engouement qu'il suscitait auprès des foules, notamment des femmes, tenait à son savoir, mais aussi à sa voix qui dégageait un charme tel qu'il fut surnommé dans les années 1970 " James Brown ", en référence à la sensualité magnétique du tube «Sex Machine» (communication personnelle du professeur Issaka Bagayogo de l'ISFRA de Bamako, 14 février 2011).

9. L'ancien Railda se situait sur l'actuel boulevard de l'Indépendance. Terrain vague plutôt que place, c'était un lieu où les enfants jouaient au football, où se tenaient les concerts et les bals populaires et où les prêcheurs trouvaient l'espace suffisant pour y réunir les foules de fidèles (communication personnelle du professeur Bagayogo, op cit.).

10. Entretien avec Haïdara du 9 juillet 2008, op. cit.

11. Ibrahima Haïdara s'est rendu célèbre pour avoir converti de nombreux paysans de la région de Sansanding dans les années 1920-1930 et surtout lutté contre la tyrannie de l'autorité coloniale déléguée en l'occurrence à Mademba Sy, le roi de Sansanding mis en place par Archinard lors de la conquête du Soudan. Cette lutte le conduira plusieurs fois en prison et il sera même déporté en Côte-d'Ivoire; voir MARTY (1920b: 183-184) et MANLEY (1997 : 324-334).

12. Kassim Traoré, «Zihara 2007 et Aid el Fitr à Tamani : Plus de 15000 fidèles originaires de 33 pays mobilisés par Haïdara », paru dans le journal Bamako Hebdo du 19 octobre 2007.

13. Il s'agit du diminutif de ce qui constitue en quelque sorte sa devise religieuse yaa bani haasimu, le "Descendant des Banû Hâshim ", le clan des Hachémites issu de l'arrièregrand-père du prophète Muhammad, Hâshim ibn Abd al-Manaf, dont les membres sont par tradition chérifs de La Mecque.

14. Tout comme le fait qu'il anime, depuis 2005, un prêche public à l'occasion du "âshûrâ » le 10 du mois de Muharram - bien qu'il s'agisse de la célébration sunnite -, ou qu'il fasse imprimer des milliers de badges sur lequel il pose avec le portrait de l'Ayatollah Khomeiny. Les relations de Haïdara avec le Centre islamique iranien de Bamako sont connues, le guide de Ançar Dine veillant d'ailleurs à inviter régulièrement des personnalités chiites maliennes lors de ses manifestations publiques. C'est d'ailleurs cet affichage qui permet à ses détracteurs de laisser entendre que Haïdara tirerait de substantielles ressources de l'Iran, pays où il a été invité une fois.

15. Voir le texte dans TRAORÉ (2007: 21-22).

16. Pour rappel, Biton Coulibaly est le nom du fondateur de l'État de Ségou. À propos de la notoriété du Super Biton de Ségou, voir notamment Florent MAZzoLENI (2008 : 43-45).

17. L'expression est employée par O. S. TRAORÉ (2007: 18) dans la traduction française de la biographie de Haïdara. 
18. Nous remercions Pierre Prud'homme, doctorant en anthropologie à l'Université de Provence, qui a rapporté cette anecdote d'un journaliste de l'ORTM rencontré lors du ziyara de novembre 2010 à Tamani.

19. Au-delà de la raillerie, qui vise à laisser entendre que l'activité religieuse de Haïdara serait de peu de valeur et même suspecte eu égard au rapport de genre sous-tendu, Haïdara s'inscrit en réalité dans une longue tradition de prêcheurs charismatiques dont le succès se mesure également à l'engouement féminin, comme en témoigne les deux prêcheurs auxquels s'est opposé Haïdara, Karamogo Lassana et Abdoul Aziz Djiré, et avec qui la compétition se situait aussi sur un principe de joute virile et de séduction à l'adresse du public féminin.

20. Pierre Prud'homme, op.cit.

21. Haïdara, entretien du 9 juillet 2008, op. cit.

22. Mais cela n'explique pas l'expression arabe ansâr al-dîn, translitérée en français par "Ançar Dine ", et l'on ignore la référence que Haïdara mobilise. Il ne s'est jamais publiquement référé au mouvement rural fondé sur un réformisme littéraliste appelé Jama'a Ansâr al-Sunna, qui s'est développé dans les années 1970 dans la région de Gao (N IEZEN 1990), pas plus qu'il ne s'est réclamé du mouvement nigérian Jamiyyat Ansâr ad-Din Naijîriyâ (REICHMUTH 1996).

23. Une lutte qui le fait, sur un tout autre registre, un héros de la révolution démocratique, ce que ne manque pas de souligner son biographe (TRAORÉ $2007: 26$ ).

24. Selon un document non daté acheté lors du Maouloud 2011 et signé d'un certain Karamoko Mamadou, prêcheur à Ségou, Haïdara aurait eu cinq épouses (dont une est décédée et une autre divorcée) et 24 enfants (dont six décédés).

25. Les données présentées ci-dessous sont issues pour l'essentiel de quatre entretiens. Les deux premiers ont été réalisés les $1^{\mathrm{er}}$ janvier 2008 et 22 février 2010 avec le prêcheur d'une section d'Ançar Dine de la région de Mopti. Les deux autres ont été effectués avec un ancien haut cadre de Ançar Dine International, le 13 juillet 2008, et avec l'un des responsables actuels, le 25 février 2011. Pour des raisons de confidentialité évidente, nous occultons ici le nom de ces personnes.

26. Cela étant, à notre connaissance, la seule association officiellement enregistrée au Mali est toujours AMSI.

27. Les nouveaux statuts et règlement de l'ADI, consultés le $1^{\text {er }}$ mars 2011, sont téléchargeables sur <http://ancardinehaidara.com/index.php? option=com_content\&view=article\&id=60:Statu\&catid=29:the-cms\&Itemid=37>.

28. Fondée en 1975, la Communauté économique des États de l'Afrique de l'Ouest regroupe les huit pays de la zone francs CFA, ainsi que le Cap Vert, la Gambie, le Ghana, la Guinée Conakry, le Liberia, le Nigeria et la Sierra Leone. Quant à l'Union économique et monétaire de l'Ouest-africain, créée en 1994, elle regroupe le Bénin, le Burkina Faso, la Côte-d'Ivoire, la Guinée-Bissau, le Mali, le Niger, le Sénégal et le Togo.

29. Il s'agit de travaux collectifs ou de marchés économiques organisés par les sections : culture et récolte, travail d'assainissement, coulage de béton, fabrication de briques, transport de sable, etc. Ces activités permettent de réunir des sommes substantielles et de mettre publiquement en scène l'abnégation de Ançar Dine capable de travailler ainsi «à cause de Dieu ", et donc pour le bien de tous. Aussi n'est-il pas rare que des gens non affiliés se joignent aux Ançars. 
30. Ce chiffre est confirmé par Haïdara lui-même dans l'article de Ousmane CouliBALY (2011).

31. Voir l'annonce de ce projet hâtivement qualifié de « banque » dans l'article de Modibo FOFANA (2010).

32. Transcription et traduction par Soumailla Camara.

33. < http://www.facebook.com/pages/Cheick-cherif-Ousmane-madanihaidara/112837645422817?v=wall>.

34. Tiré de l'article de Ben Dao du 8 mars 2010, op. cit.

35. Sur le cheikh Kishk, voir la notice biographique de Joel GORDON (2004).

36. Il avait déjà donné ce nom au second enfant de son épouse divorcée Fatoumata Traoré (information de Karamoko Mamadou, op. cit.).

37. Selon l'article de Ben Dao du 8 mars 2010 (op. cit.), l'édition 2010 du Maouloud aurait réuni 38548 participants. Le Maouloud 2011, qui semble avoir dépassé ce chiffre, s'est malheureusement terminé par la mort de 36 personnes lors d'une bousculade le 21 février. L'enquête est en cours, mais la responsabilité des services publics paraît engagée, eu égard au sous-effectif criant des forces de police et à l'accès dans un stade prévu pour accueillir 25000 personnes.

38. Texte reproduit du calendrier de 2005 que Ançar Dine commercialise.

39. Sahîh Al-Bukhârî, Kitâb Manâqib al-Ansâri [Livre des Mérites des Ansar], Bâb [chapitre] «Wufûdil Ansâri Ilan-Nabiyyi Bi Makkata Wa Baatil Aqabah ».

40. Al Hajj Umar al Futi, les «Rimah », 1973, t. 1, chapitre 23, p. 175, cité par Madina LY-TALL (1991 : 140).

\section{RÉSUMÉS}

Résumé

Ce texte analyse le mouvement islamique Ançar Dine fondé en 1991 par Chérif Ousmane Madani Haïdara, un personnage charismatique qui a bouleversé les modalités d'expression de l'islam dans l'espace public malien. Ançar Dine est une organisation dont la forme hybride oscille entre association de la société civile, ONG caritative, confrérie intramondaine et internationalisme fédéraliste. Comptant plus de 70000 membres, essentiellement au Mali, en Côte-d'Ivoire et au Burkina Faso, Ançar Dine est devenue un espace de critiques à l'égard de l'establishment et la principale force d'opposition à la réforme sunnite communément appelée « wahhabite ». Il prône un réformisme « africain » et une confirmation islamique fondée sur le serment coranique appelé bay'a. Travaillé par une tension croissante entre le charismatique et le bureaucratique, Ançar Dine vise en final à mettre en place un espace d'autonomie spirituelle, sociale, économique et politique qui se fonde sur une « raison populiste ».

Cherif Usmane Madane Haydara and the Islamic Movement Ançar Dine

In this text I analyze the Islamic movement Ançar Dine, established in 1991 by Cherif Usmane Madane Haydara, a charismatic figure who transformed the modalities of expressions of Islam in 
the Malian public sphere. Ançar Dine is a hybrid organization, which can be situated between the association of civil society, the charitable NGO, the intra-worldly brotherhood, and the federalist internationalism. With more than 70.000 members, mainly in Mali, Ivory Coast and Burkina Faso, Ançar Dine is a critical platform against the establishment and the principal opposition force against the Sunnite Reform commonly called "Wahhabite". It advocates an "African" reformism and an Islamic confirmation based on a koranic pledge called bay'a. Despite an increasing tension between the charismatic and bureaucratic powers on the organization, Ançar Dine aims at a spiritual, social, economic and political autonomy sphere, based on a "populist reason".

INDEX

Mots-clés : Mali, Chérif Ousmane Madani Haïdara, Ançar Dine, autonomie, islam populaire, réformisme islamique

Keywords : Mali, Cherif Usmane Madane Haydara, Ançar Dine, Autonomy, Popular Islam, Islamic Reformism

\section{AUTEUR}

\section{GILLES HOLDER}

CNRS ; Centre d'études africaines, EHESS-IRD (UMR 194), Paris. 